\title{
A FAST CONTINUATION SCHEME FOR ACCURATE TRACING OF NONLINEAR OSCILLATOR FREQUENCY RESPONSE FUNCTIONS
}

by

\author{
Guoqiang Chen ${ }^{1,2}$ and J F Dunne ${ }^{2 *}$ \\ 1Department of VehicleEngineering \\ The School of Mechanical and Power Engineering \\ Henan Polytechnic University, Jiaozuo, 454003, China \\ 2Department of Engineering and Design \\ The School of Engineering and Informatics \\ The University of Sussex, Falmer, Brighton, BN1 9QT, UK.
}




\begin{abstract}
A new algorithm is proposed to combine the split-frequency harmonic balance method (SFHBM) with arc-length continuation (ALC) for accurate tracing of the frequency response of oscillators with non-expansible nonlinearities. ALC is incorporated into the SF-HBM in a two-stage procedure: Stage I involves finding a reasonably accurate response frequency and solution using a relatively large number of low-frequency harmonics. This step is achieved using the SF-HBM in conjunction with ALC. Stage II uses the SF-HBM to obtain a very accurate solution at the frequency obtained in Stage I. To guarantee rapid path tracing, the frequency axis is appropriately subdivided. This gives high chance of success in finding a globally optimum set of harmonic coefficients. When approaching a turning point however, arc-lengths are adaptively reduced to obtain a very accurate solution. The combined procedure is tested on three hardening stiffness examples: a Duffing model; an oscillator with non-expansible stiffness and single harmonic forcing; and an oscillator with nonexpansible stiffness and multiple-harmonic forcing. The results show that for non-expansible nonlinearities and multiple-harmonic forcing, the proposed algorithm is capable of tracingout frequency response functions with high accuracy and efficiency.
\end{abstract}

Keywords: nonlinear; oscillator; fast tracing; frequency; response.

22 main-section pages (double spaced) 38 references Figures 1 - 16 No appendices 


\section{INTRODUCTION}

Nonlinear oscillator models represent a wide class of dynamic systems in many areas of physics and engineering [1-3]. For example, the classic Duffing model for mechanical systems [4, 5], the delayed-reaction model for sensors and actuators [6], the time-varying mass model for crane and bridge cables [7], the M-shaped bent-beam model [8], and the coupled linear-nonlinear oscillator model [9-11]. Nonlinear oscillator models are useful for revealing unexpected internal system behaviour associated with nonlinear phenomena, for predicting resonances in forced motion, and for refining system designs in engineering applications. The analysis of nonlinear oscillators can be complicated by the sensitivity of responses to relatively small changes in system parameters, excitation intensities, and initial conditions. Small parameter changes can cause for example, a transition from periodic behavior to chaos, or stable motion to instability. Periodic solutions and frequency response functions are of particular interest, having received considerable attention [12-16]. Periodic solutions expressed in the form of finite Fourier series are generally difficult to obtain in closed-form except for very simple oscillators [17] or special cases [18-19]. Accurate closedform expressions are difficult to obtain [20-21], which is one reason why great emphasis has been placed on analytical approximations such as the perturbation method [22], multiple scales [23-24], and the Harmonic Balance Method (HBM) [17][25-28].

The HBM is effective in handling models with strong 'expansible' nonlinearities, making it by far the most widely-used method [17][25-26][28-29]. By truncating a Fourier series, then expanding and simplifying the oscillator differential equation into trigonometric components, algebraic balance equations are obtained to find the undetermined harmonic coefficients. The balance equation system is then solved to obtain the approximate response. A nonlinear polynomial model for stiffness (i.e. with $5^{\text {th }}$ degree or higher) is generally non-expansible, with the result that an explicit closed-form set of algebraic balance equations is extremely difficult to construct. An alternative numerical approach to handle non-expansible 
nonlinearities is to use the Fast Fourier transform (FFT) to implicitly construct the balance

equations. In general non-expansible nonlinearities arise when it is not practical to construct (in closed-form) the algebraic equations needed to implement the HBM. This type of nonlinearity occurs where it is difficult to express the Fourier coefficients of a nonlinear function of the response in terms of the Fourier coefficients of the response itself. Consider for example, an integer power nonlinearity. It is possible to write down a general recursive relationship [30] to obtain the Fourier coefficients for $y^{m}$ in terms of the Fourier coefficients of $y$ (for any integer $m$ ). Quadratic and cubic terms i.e. for $m=2$ and 3 , are indeed expansible but for higher powers the expansions become impractical. Moreover, for any nonlinear function more complicated than a simple power, constructing even a recursive relationship is generally not possible. Nonlinear functions are then deemed to be totally nonexpansible.

For multi-harmonic forcing and high degree nonlinearities, a large number of solution harmonics are generally needed. By using FFT generated algebraic equations, any number of response harmonics can be included, enabling error reduction to any desired level. But a solution involving a huge number of harmonics is a serious burden when tracing across the frequency domain, resulting in unacceptably high computational cost.

Arc-Length Continuation (ALC) is a well-known method for solving boundary value problems. ALC in conjunction with the HBM [14][27][31-33] has also been used to improve the analysis of nonlinear oscillators. A conventional ALC scheme first involves finding the unknown response frequency and response harmonics. This is achieved by augmenting the system of algebraic balance equations with an ALC equation [14]. The augmented system is solved using a known solution at a particular frequency plus a specified arc-length. This generates a new frequency and a corresponding set of approximate solution harmonics. Repeated application traces out the entire solution across the frequency range. The tracing procedure continues using the current solution as the starting guess for the computations 
involving the subsequent arc length. The solution accuracy at each step is critical to the

success of a traced solution in a subsequent step, especially around turning points. An inaccurate solution at a particular step can result in failure to find a solution around a turning point. Moreover, to undertake stability analysis, an accurate Jacobian is needed and this depends on having an accurate response solution [14-15]. ALC has been effectively used in conjunction with the classical HBM [33] to detect tangent and flip bifurcations of limit cycles. In general, this level of accuracy can only be achieved by including an adequate number of harmonics in the response.

The split-frequency harmonic balance method (SF-HBM) [15], and an associated stability analysis method [16], were proposed to obtain Period-1 and sub-harmonic responses of nonlinear oscillators. In this method, the equation error is defined in terms of two functions [34], which split the Fourier series solution into a low-frequency group and a high-frequency group. The low-frequency group, which starts off with the mean and fundamental and gradually adds more terms, is obtained using a conventional HBM. In a separate step, the high-frequency group is approximated from the low frequency group by iteration. By progressively increasing the number of harmonics in the low-frequency group, the accuracy of the total solution (which can include a very large number of harmonics) can be dramatically improved. One benefit of the method is that it can handle oscillators with nonexpansible nonlinearities and multi-harmonic forcing with excellent accuracy and efficiency. Like all HBM approaches however, the success of the SF-HBM depends on making a good initial guess for the amplitude and phase of the fundamental component. An algorithm that can make full use of the accuracy of the SF-HBM to efficiently trace out frequency response functions is clearly of great benefit.

Elsewhere, to improve the accuracy and efficiency of the HBM, two very different methods have been combined with the classical HBM. For example, by removing solution harmonics with insignificant coefficients, the so called 'harmonic selection technique' [35] can be quite 
effective (compared with the classical HBM) in reducing the computation time by up to $50 \%$.

The 'homotopy harmonic balance method' [36-37] (which combines a parameter expansion with the HBM) can be effective in obtaining steady state responses of strongly nonlinear oscillators such as the Duffing equation [36]. A novel iterative homotopy harmonic balancing method has also been proposed offering high accuracy, efficiency, and simplicity in obtaining periodic solutions of a strongly nonlinear oscillator of the form $\ddot{y}+\alpha+\beta y^{3}+\gamma y^{1 / 3}=0$ [37].

Software packages such as AUTO-07 and MATCONT are open-source codes widely-used for numerical bifurcation analysis of a general class of dynamic systems, in particular for exploiting continuation to compute equilibrium points and periodic solutions of autonomous ordinary differential equations. There are however some reported difficulties in using (Fortran-coded) AUTO-07 to continue periodic solutions of the Duffing oscillator for particular parameter values. By contrast the more recent (Matlab-coded) MATCONT software can obtain periodic solutions of forced oscillator equations provided they are converted to (higher order) autonomous systems. However to successfully undertake bifurcation analysis for a pair of coupled oscillators using MATCONT, it is reported in [38] that for certain parameter values, the computational cost becomes prohibitive.

In this paper, a new algorithm is developed and tested, by combining the SF-HBM with arclength continuation. Special measures are needed to exploit the iterative nature of the SFHBM, which does not readily lend itself to extension to an ALC scheme. To overcome this problem, the SF-HBM and ALC are combined into a two-stage 7-step iterative scheme. The objective of the paper is to construct the computational basis of the proposed algorithm, and to verify the computational accuracy and efficiency via three numerical examples. Compared with the other analytical methods, for example, the perturbation method and multiple scales, the proposed approach offers two prominent features. First it is totally independent of any small parameter assumption. And second, for arbitrarily strong nonlinear oscillators, the proposed method is guaranteed to obtain a solution with any degree of accuracy. 


\section{ADAPTING THE SF-HBM FOR USE WITH ARC-LENGTH CONTINUATION}

Here a brief outline is given of the original SF-HBM [15] and how this is adapted for use with arc-length continuation. The computational details of the combined SF-HBM ALC algorithm are given in Section 3. The SF-HBM can be used to obtain a very accurate frequency response for a SDOF oscillator model of the form:

$$
\ddot{y}+F(y, \dot{y})=f(t)
$$

where $F(y, \dot{y})$ is in general a strongly nonlinear function. The periodic excitation $f(t)$ is represented as a Fourier series with multi-harmonics, fundamental frequency $\Omega_{1}$, and coefficients $C_{k}$ as follows:

$$
f(t)=\sum_{k=-m}^{m} C_{k} \mathrm{e}^{j \Omega_{k} t}
$$

A periodic response $y$ is assumed, with the mean $A_{0} / 2$, and harmonic amplitudes $A_{m}$, and phases $\phi_{m}$, written as a Fourier series:

$$
y(t)=\frac{A_{0}}{2}+\sum_{m=1}^{\infty} A_{m} \cos \left(m \Omega_{1} t+\phi_{m}\right)
$$

Equation (3) starts with similar assumptions as the approximate analytical methods [17][2528]. The SF-HBM [15] splits the periodic response into a low-frequency harmonic group and a high-frequency group. The low-frequency group (with suffix $L F$ ) comprises the mean value $A_{0} / 2$ and $k$ low-frequency harmonics:

$$
y_{L F}(t)=\frac{A_{0}}{2}+\sum_{m=1}^{k} A_{m} \cos \left(m \Omega_{1} t+\phi_{m}\right)
$$

The high-frequency group (with suffix $H F$ ) includes the remaining harmonics such that:

$$
y_{H F}(t)=\sum_{m=k+1}^{\infty} A_{m} \cos \left(m \Omega_{1} t+\phi_{m}\right)
$$

The iterative way of obtaining an accurate solution in the SF-HBM, starts with the lowfrequency group including just the mean value $A_{0} / 2$ and the first harmonic i.e.: 


$$
y_{L F}(t)=\frac{A_{0}}{2}+A_{1} \cos \left(\Omega_{1} t+\phi_{1}\right)
$$

The coefficients in the high frequency group in equation (5) are approximated by iteration using the low frequency group. In a series of steps, high-frequency harmonics are transferred to the low-frequency group. The scheme makes use of the equation error associated with trial solution equation (3) being substituted into equation (1) as follows:

$$
e(t)=\ddot{y}+F(y, \dot{y})-f(t)
$$

To simplify construction of the usual HBM algebraic equations, the SF-HBM uses an FFT of the equation error $e(t)$ which is discretized at $N$ time intervals of duration $T / N$ spanning the period $T$. The FFT of the equation error then becomes:

$$
E(K)=\sum_{m=1}^{N} e\left(t_{m}\right) \mathrm{e}^{-j 2 \pi K(m-1) / N}, 0 \leq K \leq N-1
$$

To construct the system of $2 k+1$ HBM algebraic equations in $2 k+1$ unknowns, the amplitudes and phases of the harmonic components in the low frequency group are set to zero. The $2 k+1$ unknown coefficients are found using a least square error solver (such as the Matlab function Isqnonlin) where the objective function constructed from the corresponding system of HBM algebraic equations can be written as:

$$
g_{k}\left(A_{0}, A_{1}, \phi_{1}, A_{2}, \phi_{2}, \ldots, A_{k}, \phi_{k}, \Omega_{1}\right)=\sum_{K=0}^{k}|E(K)|^{2}=\sum_{K=0}^{k}\left|\sum_{m=1}^{N} e\left(t_{m}\right) e^{-j 2 \pi K(m-1) / N}\right|^{2}
$$

The least square solution methodology using Isqnonlin for example, also supplies an estimate of the Jacobian which is useful for stability analysis. The objective function equation (9) is written explicitly to show the fundamental frequency $\Omega_{1}=1 / T$ as a parameter. When using the SF-HBM alone, the period $T$ would be known, therefore it is not necessary to solve for $\Omega_{1}$. Equation (9) would thus not normally include $\Omega_{1}$ as a parameter. But in using the ALC method it is necessary to find the frequency for a specific arc length. Thus objective function equation (9) is written in a form appropriate for adaptation to ALC as now explained. 


\section{Adapting the SF-HBM}

The traditional HBM, implemented using Equation (9), can be rewritten as:

$$
g_{k}\left(A_{0}, A_{1}, \phi_{1}, A_{2}, \phi_{2}, \ldots, A_{k}, \phi_{k}, \Omega_{1}\right)=0
$$

In order to trace along a solution branch, a continuation parameter $\alpha$ is introduced into Equation (10). From Equation (10), we can get:

$$
\frac{\partial g_{k}}{\partial A_{0}} \frac{\mathrm{d} A_{0}}{\mathrm{~d} \alpha}+\sum_{m=1}^{k}\left(\frac{\partial g_{k}}{\partial A_{m}} \frac{\mathrm{d} A_{m}}{\mathrm{~d} \alpha}+\frac{\partial g_{k}}{\partial A_{m}} \frac{\mathrm{d} \phi_{m}}{\mathrm{~d} \alpha}\right)+\frac{\partial g_{k}}{\partial \Omega_{1}} \frac{\mathrm{d} \Omega_{1}}{\mathrm{~d} \alpha}=0
$$

The tangent vector $\left[A_{0}, A_{1}, \phi_{1}, A_{2}, \phi_{2}, \ldots, A_{k}, \phi_{k}, \Omega_{1}\right]$ can be normalized by the unit length to give:

$$
\left(\frac{\mathrm{d} A_{0}}{\mathrm{~d} \alpha}\right)^{2}+\sum_{m=1}^{k}\left[\left(\frac{\mathrm{d} A_{m}}{\mathrm{~d} \alpha}\right)^{2}+\left(\frac{\mathrm{d} \phi_{m}}{\mathrm{~d} \alpha}\right)^{2}\right]+\left(\frac{\mathrm{d} \Omega_{1}}{\mathrm{~d} \alpha}\right)^{2}=1
$$

from which, the square of the total differential of $\alpha$ is found to satisfy the equation:

$$
(\mathrm{d} \alpha)^{2}=\left(\mathrm{d} A_{0}\right)^{2}+\sum_{m=1}^{k}\left\{\left(\mathrm{~d} A_{m}\right)^{2}+\left(\mathrm{d} \varphi_{m}\right)^{2}\right\}+\left(\mathrm{d} \Omega_{1}\right)^{2}
$$

By writing the excitation frequency $\Omega_{1}$ as an explicit variable, the proposed combination of the SF-HBM with ALC starts by introducing a (small) arc-length $s$, defined as:

$$
\begin{aligned}
s & =\mathrm{d} \alpha \\
& =\sqrt{\left(\mathrm{d} A_{0}\right)^{2}+\sum_{m=1}^{k}\left\{\left(\mathrm{~d} A_{m}\right)^{2}+\left(\mathrm{d} \varphi_{m}\right)^{2}\right\}+\left(\mathrm{d} \Omega_{1}\right)^{2}} \\
& =\sqrt{\left(A_{0}-A_{0 \mathrm{p}}\right)^{2}+\sum_{m=1}^{k}\left\{\left(A_{m}-A_{m \mathrm{p}}\right)^{2}+\left(\phi_{m}-\phi_{m \mathrm{p}}\right)^{2}\right\}+\left(\Omega_{1}-\Omega_{\mathrm{lp}}\right)^{2}}
\end{aligned}
$$

where $A_{0 \mathrm{p}}, A_{1 \mathrm{p}}, \phi_{\mathrm{lp}}, A_{2 \mathrm{p}}, \phi_{2 \mathrm{p}}, \cdots, A_{k \mathrm{p}}, \phi_{k \mathrm{p}}$ represent the vector of parameters obtained at the previous frequency $\Omega_{1 \mathrm{p}}$. By setting the arc length to a reasonably small value $s_{\text {set }}$ within the iteration, an additional equation is created and added to the system of simultaneous algebraic HBM equations. The complete system of equations, including the arc-length equation, then becomes: 
An arc length term is now added to equation (9) to create modified objective function:

$$
\begin{aligned}
g_{\mathrm{A} k}\left(A_{0}, A_{1}, \phi_{1}, A_{2}, \phi_{2}, \cdots, A_{k}, \phi_{k}, \Omega_{1}\right) & =g_{k}\left(A_{0}, A_{1}, \phi_{1}, A_{2}, \phi_{2}, \cdots, A_{k}, \phi_{k}, \Omega_{1}\right)+\left|s-s_{\text {arc }}\right|^{2} \\
& =\sum_{K=0}^{k}\left|\sum_{m=1}^{N} e\left(t_{m}\right) \mathrm{e}^{-j 2 \pi K(m-1) / N}\right|^{2}+\left|s-s_{\text {set }}\right|^{2}
\end{aligned}
$$

Within the SF-HBM [15], the high-frequency group is updated using the following equation:

$$
\ddot{y}_{H F}+\Omega_{1}^{2} y_{H F}=-\frac{A_{0} \Omega_{1}^{2}}{2}+\Omega_{1}^{2}\left(y_{L F}+y_{H F}\right)-F\left(y_{L F}+y_{H F}, \dot{y}_{L F}+\dot{y}_{H F}\right)+f(t)+e\left(y_{L F}+y_{H F}\right)
$$

Because the first harmonic is not included in the high frequency group, neither side of equation (17) contains any fundamental frequency component. Equation (17) can therefore be written as:

$$
\begin{aligned}
& -\frac{A_{0} \Omega_{1}^{2}}{2}+\Omega_{1}^{2}\left(y_{L F}+y_{H F}\right)-F\left(y_{L F}+y_{H F}, \dot{y}_{L F}+\dot{y}_{H F}\right)+f(t)+e\left(y_{L F}+y_{H F}\right) \\
& =\frac{a_{0}}{2}+\sum_{n=2}^{\infty} a_{n} \cos \left(n \Omega_{1} t\right)+b_{n} \sin \left(n \Omega_{1} t\right)
\end{aligned}
$$

The high frequency group $y_{H F}$ can be expressed in terms of the low-frequency group, and solved (in the previous iteration) as the particular integral solution of equation (17). To be specific, the high-frequency group updating equation is given as:

$$
y_{H F u}=-\frac{1}{\Omega_{1}^{2}}\left\{\sum_{n=k+1}^{\infty} \frac{1}{n^{2}-1}\left\{a_{n} \cos \left(n \Omega_{1} t\right)+b_{n} \sin \left(n \Omega_{1} t\right)\right\}\right\}
$$

Therefore, the total updated solution to the oscillator model can be written as: 


$$
\begin{aligned}
y_{u}(t) & =\left\{\frac{A_{0}}{2}+A_{1} \cos \left(\Omega_{1} t+\phi_{1}\right)+A_{2} \cos \left(2 \Omega_{1} t+\phi_{2}\right)+\cdots+A_{k} \cos \left(k \Omega_{1} t+\phi_{k}\right)\right\} \\
& -\frac{1}{\Omega_{1}^{2}}\left\{\sum_{n=k+1}^{\infty} \frac{1}{n^{2}-1}\left\{a_{n} \cos \left(n \Omega_{1} t\right)+b_{n} \sin \left(n \Omega_{1} t\right)\right\}\right\}
\end{aligned}
$$

\section{THE CHALLENGES TO SUCCESSFUL COMBINATION OF THE SF-HBM WITH ALC}

Four issues need to be overcome to successfully combine the SF-HBM with ALC so that an accurate nonlinear oscillator frequency response can be rapidly traced across the entire frequency. Here these issues are explained. The first issue relates to finding the fundamental frequency, and the coefficients in equation (16) using a least square minimization method. The objective function equation (16) does not readily allow a minimum to be found. This is shown using figure 1 where a current solution is designated at the point $\mathrm{S}_{q}$, and the next expected solution at the point $S_{q+1}$. In the least square solver, the lower and upper boundaries of frequency $\Omega_{1}$ are set to $\Omega_{1 q}$ and $\Omega_{1 q}+\Delta \Omega_{1}$ respectively. The worst cases of the solver output can be divided into two categories: i) the optimization fails because an unacceptable local optimum solution is obtained; or ii) the path tracing procedure cannot proceed because the globally optimal solution is obtained at the frequency $\Omega_{1 q}$ (for example when the true solution is $\mathrm{S}_{q}$ and the false solutions are $\mathrm{S}_{\mathrm{F} q 1}$ or $\mathrm{S}_{\mathrm{F} q 2}$ ). Although the former case is a common problem in nonlinear optimization, the strong nonlinearity of equation (16) exacerbates the problem. The latter case results from the SF-HBM starting the lowfrequency group with just a mean and a single harmonic that poorly approximates the true solution - a more serious problem for multi-harmonic forcing.

The second issue relates to turning points around which the optimization of equation (16) fails, and the path tracing procedure cannot proceed. This problem, and the reason for it, can also be explained using figure 1. The objective function equation (16) comprises the conventional SF-HBM terms, and an (arc-length) continuation term. If at each continuation step, the correct solution is obtained for the coefficients associated with the low-frequency 
group, then both terms in equation (16) are effectively zero. As the tracing procedure approaches a turning point, for example $\mathrm{P}_{1}$, when the current solution is at the point $\mathrm{S}_{n}$, then the next correct solution should be on the surface of a $2 k+1$ dimensional half-hypersphere with the center $\mathrm{S}_{n}$ and radius $s_{\text {set }}$. The half-hypersphere surface is shown as a semi-circular arc in figure 1. Because the conventional SF-HBM terms are zero, a correct solution to equation (9) is obtained necessarily on the frequency response curve. For the case shown in figure 1, there is evidently no intersection point of the half arc and the response curve. Consequently convergence of the least square solver would not occur. Moreover, even if a turning point is accurately found, it may still be difficult to trace the next solution without any prior knowledge of the system response. When the curve segment $\mathrm{P}_{1} \mathrm{P}_{2}$ is traced by starting from the current solution $\mathrm{P}_{1}$, continuation may converge to a solution near to the previous solution $\mathrm{S}_{n}$ but it is very unlikely to be equal to the point $\mathrm{S}_{n}$ because the low-frequency group is only an approximation to the full solution.

The third issue is that traditional continuation does not appear to incorporate the SF-HBM easily. Equation (16) is a complicated implicit function of the low-frequency harmonics - its value is highly dependent on the iteration number and starting solutions i.e. in figure 1: $S_{001}$ and $\mathrm{S}_{002}$ for the segment $\mathrm{S}_{001} \mathrm{P}_{1} ; \mathrm{S}_{101}$ and $\mathrm{S}_{102}$ for the segment $\mathrm{S}_{101} \mathrm{P}_{2}$; and $\mathrm{S}_{201}$ and $\mathrm{S}_{202}$ for the segment $\mathrm{P}_{1} \mathrm{P}_{2}$. The fact that the conventional SF-HBM does not guarantee that the equation error would continue to reduce with each iteration complicates the objective function.

The fourth issue relates to the speed of convergence and the accuracy of computation. Since the continuation term in objective function equation (16) is a strongly nonlinear function of its arguments (i.e. the magnitudes, phases, and the fundamental frequency), it has at least one locally optimum solution. This considerably increases the difficulty of finding a globally optimal solution especially for the case where more harmonics are needed in the 
total solution for multi-harmonic forcing. More computation time therefore is needed.

Furthermore, equation (17) is derived from two Mickens functions [34] with fixed fundamental frequency that actually needs to become a variable in any merged SF-HBMALC scheme. This adds a new error source to equation (17) which reduces computational efficiency. Setting the equation error terms to zero in equations (17) and (18) allows successive iterations to reduce the magnitude of the equation error but without modification, considerable computation time is needed to achieve acceptable accuracy.

\section{THE COMPUTATIONAL DETAILS OF THE COMBINED SF-HBM ALC ALGORITHM}

Measures are now proposed to address the four issues discussed in Section 3. Adoption of these measures then culminates in the detailed description of a new 7-step procedure for fast tracing of frequency response functions.

The first issue is addressed by dividing the frequency steps $\Delta \Omega_{q}$ into $J$ intervals in the optimization process within ALC, shown by a ' + ' or '-' sign in figure 1, corresponding respectively to subdivision of a step $\left[\Omega_{q}, \Omega_{q}+\Delta \Omega_{1}\right]$ tracing in a positive direction, or a step $\left[\Omega_{q}-\Delta \Omega_{1}, \Omega_{q}\right]$ tracing in a negative direction. The optimization interval width $\Delta \Omega_{1}$ should satisfies the inequality:

$$
\Delta \Omega_{1} \leq s_{\text {set }}
$$

where the subintervals are defined as:

$$
\left[\Omega_{q}+(j-1) \delta_{\mathrm{S}}, \Omega_{q}+j \delta_{\mathrm{S}}\right] \text { or }\left[\Omega_{q}-j \delta_{\mathrm{s}}, \Omega_{q}-(j-1) \delta_{\mathrm{S}}\right], j=1,2,3, \cdots, J
$$

and where $\delta_{\mathrm{S}}=\Delta \Omega_{1} / J$, and $J$ is the number of optimal fundamental frequencies at $\Omega_{1 j}$. Solutions $X_{k j}, j=1,2,3, \cdots, J$ can be found using a least square solver in the $j^{\text {th }}$ subinterval; the corresponding $j^{\text {th }}$ errors are designated as $e_{k j}$. A reasonable error limit $e_{\lim }$ is selected to control the progress of the least square solver. The (optimal) fundamental frequency, and solution harmonics, are determined from the equation: 


$$
\left(\Omega_{q}, X_{q}\right)=\left(\Omega_{1 i}, X_{q i}\right), \quad i=\left\{\begin{array}{l}
\max \left\{j \mid e_{q j} \leq e_{\mathrm{lim}}, j=1,2,3, \cdots, J\right\} \\
\max \left\{j \mid e_{q j}>e_{\mathrm{lim}}, e_{q j}=\min \left\{e_{q j}\right\}, j=1,2,3, \cdots, J\right\}
\end{array}\right.
$$

The measures adopted in equations $(22)-(23)$ guarantee that the path tracing process will proceed despite possible failure to find the globally optimal solution. Thus, although finding the globally optimal solution is not guaranteed, the chances of finding it significantly improve with increasing $J$.

The second issue is addressed by recognizing that for the low-frequency group, if the least square error solver fails to find a solution with reasonable accuracy, it must be concluded that a turning point is being approached very closely. Since, as shown in figure 1 , a true solution necessarily occurs at an intersection between the half arc and the response curve, a true solution can only be found when there is at least one intersection point. In such circumstances the arc-length $s_{\text {set }}$ should be reduced and the optimization procedure repeated. With an increasing number of iterations, the interval width in equation (21) becomes progressively smaller. This process continues until a reasonably accurate solution is found.

As to the path tracing around the turning point, each segment is traced separately. The first segment is traced in the positive $(+)$ direction from a sufficiently low frequency $\Omega_{L}$. For example, the segment $\mathrm{S}_{001} \mathrm{P}_{1}$ in figure 1 , and the first turning point frequency $\Omega_{\mathrm{P} 1}$, with corresponding solution, are obtained. The second segment is in the negative (-) direction from a sufficiently high frequency $\Omega_{H}$, for example, the segment $S_{101} P_{2}$ in figure 1 , where the second turning point at frequency $\Omega_{\mathrm{P} 2}$ is obtained. The new starting points, neither of which are on the first or second segment in the frequency interval (i.e. $\left[\Omega_{\mathrm{P} 2}, \Omega_{\mathrm{P} 1}\right]\left(\Omega_{\mathrm{P} 2}<\Omega_{\mathrm{P} 1}\right)$ or $\left.\left[\Omega_{\mathrm{P} 1}, \Omega_{\mathrm{P} 2}\right]\left(\Omega_{\mathrm{P} 1}<\Omega_{\mathrm{P} 2}\right)\right)$, are selected using the standard form of the SF-HBM with random initial guesses.

Additional efficiency improvements can be obtained by first noting (for the Duffing oscillator 
for example), that shifting the phase of a high magnitude stable solution by $-90^{\circ}$, gives an unstable high magnitude solution [15]. This enables new segments and turning points to be obtained. Second, since the new starting points $S_{201}$ and $S_{202}$ shown in figure 1, and the new path tracing process is continued in two directions, if the arc-length between any two turning points is less than the specified error, they can be considered as one turning point. This process can continue until all turning points and segments are found, enabling all of the segments to form a continuous curve.

The fourth issue can be addressed in two-stages as follows: the first stage is to find a reasonably accurate fundamental frequency and solution up to a relatively large number of low-frequency harmonics using the standard SF-HBM in conjunction with arc-length continuation. The second stage is to find a very accurate solution using the solution found (at the first stage) as the starting choice using the conventional SF-HBM at the fundamental frequency found by iteration.

\section{A 7-step algorithm combining the SF-HBM and ALC}

When the measures discussed previously to address the four problem issues are implemented using the SF-HBM and ALC, the procedure can be described in the form of a 7-step algorithm as shown in figure 2. Three control parameters are used in the algorithm as follows: First there is an outer-loop variable ' $q$ ' which represents the 'serial' number of the tracing solution points for each segment of the frequency response curve. Then there is the arc-length factor $\lambda$ which is used to control the current arc-length i.e. $s_{\mathrm{set}}=\lambda s_{0}$. And the third parameter is $K_{\text {switch }}$ which in stage I, represents the maximum number of the harmonics included in the low frequency group. The step-by-step details are now explained.

Step 1: Select the parameters for the outer-loop in figure 2 and select accurate starting values for the vector of amplitudes and phases: $X_{-1}=\left[A_{0-1}, A_{1-1}, \phi_{1-1}, A_{2-1}, \phi_{2-1}, \cdots, A_{N / 2-1}\right.$, 
$\left.\phi_{N / 2-1}\right]$ and $X_{0}=\left[A_{00}, A_{10}, \phi_{10}, A_{20}, \phi_{20}, \cdots, A_{N / 20}, \phi_{N / 20}\right]$ using the conventional SF-HBM, at two

specific fundamental frequencies $\Omega_{1-1}$ and $\Omega_{10}$, then compute the arc-length $s_{0}$ using equation (14) based on the two starting solutions; set the arc-length factor to $\lambda=1$, and set the 'serial' number $q$ for the following solution points to $q=1$.

Step 2: Select the parameters for the middle- and inner-loops. Start the iteration at $i=1$ and $k=1$. Set $y_{H F 0}(t)=0$. The initial guesses for $A_{0}, A_{1}, \phi_{1}$, and for the least square solver, are set using the linear extrapolation as follows:

$$
\left\{\begin{array}{l}
\Omega_{1 \mathrm{~g}}=(1+\lambda) \Omega_{1 q-1}-\lambda \Omega_{1 q-2} \\
{\left[A_{0 \mathrm{~g}}, A_{1 \mathrm{gg}}, \phi_{1 \mathrm{~g}}\right]=(1+\lambda)\left[A_{0 q-1}, A_{1 q-1}, \phi_{1 q-1}\right]-\lambda\left[A_{0 q-2}, A_{1 q-2}, \phi_{1 q-2}\right]}
\end{array}\right.
$$

Step 3: Compute the amplitudes and phases associated with the low frequency group. Set the current arc-length $s_{\mathrm{set}}=\lambda s_{0}$, then express the solution of equation (1) as:

$$
y_{i}=\left(A_{0 i} / 2\right)+A_{1 i} \cos \left(\Omega_{1} t+\phi_{1 i}\right)+A_{2 i} \cos \left(2 \Omega_{1} t+\phi_{2 i}\right)+\cdots+A_{k i} \cos \left(k \Omega_{1} t+\phi_{k i}\right)+y_{H F i}
$$

Solve for $A_{0 i}, A_{1 i}, A_{2 i}, \ldots, A_{k i}$ and $\phi_{1 i}, \phi_{2 i}, \ldots, \phi_{k i}$ via a $k$-term HBM solution. The amplitudes and phases associated with the low frequency group are actually obtained from an unconstrained least-square-sum associated with the objective function equation (16). If $k \leq K_{\text {switch }}$ then equation (17) should be used, i.e. use the SF-HBM with ALC, where the (optimum) fundamental frequency $\Omega_{1 q i}$ can be obtained using equations (21) - (23). But if $k>K_{\text {switch }}$ then equation (9) should be used i.e. the standard form of SF-HBM.

Step 4: Compute the high-frequency group. Obtain the Fourier components of equation (18) with the equation error term omitted using the predicted fundamental frequency $\Omega_{1 q i}$. And with coefficients $a_{n}$ and $b_{n}$, obtain an update estimate $y_{H F i}$ for the high frequency group using Equation (19).

Step 5: Increase $i$ by 1 to $i=i+1$, and choose $X_{q i}$ as the initial guess for the coefficients associated with the low-frequency group for the next iteration; go back to Step 3 if the 
maximum number of iterations $I_{\max }$ for the low-frequency group is not reached (i.e. $\left.i \leq I_{\max }\right)$;

By contrast, if the maximum number of iterations is reached (i.e. $i>I_{\max }$ ), go to Step 6 .

Step 6: Introduce an additional low-frequency harmonic to the low-frequency group i.e. set $y_{H F 0}=y_{H F i}, i=1$; increase $k$ by 1 to $k=k+1$; and set $\left[X_{q i}, A_{q k i}, \phi_{q k i}\right]$ (in which $A_{q k i}$ and $\phi_{q k i}$ are the initial guesses for the next iteration, of the amplitude and phase corresponding to the lowest harmonic in equation (19)) then go to Step 3 if the maximum number $K_{\max }$ of harmonics for the low-frequency group is not reached (i.e. $k \leq K_{\max }$ ); But if the maximum number is reached (i.e. $k>K_{\max }$ ), then go to Step 7 .

Step 7: Equation error evaluation: Two scenarios are defined to guide selection at this step: Scenario-1: The equation error is unacceptable (i.e. larger than the pre-set error limit). If the arc-length factor $\lambda$ is small enough (i.e. smaller than the pre-set limit), the previous solution is a turning point, and calculation should be stopped. Alternatively, if the arc-length factor $\lambda$ is multiplied by a value less than 1 , for example $\lambda=\lambda / 2$, then go to Step 2 . Scenario2: The equation error is acceptable (i.e. smaller than the pre-set error). For this scenario, store the frequency, amplitudes, and phases obtained (i.e. $\Omega_{1 q}$ and $X_{q}$ ), then set $\lambda=1$, and increase $q$ by 1 to $q=q+1$, and go to Step 2 .

\section{TESTING THE COMBINED SF-HBM ALC SCHEME}

In what follows, the computational merits and advantages of the proposed 7-step algorithm are verified and demonstrated using three numerical examples, namely a Duffing model with single harmonic forcing, a SDOF oscillator with a $9^{\text {th }}$ degree nonlinearity and single harmonic forcing, and the same oscillator with 24-harmonic periodic forcing. Regarding the numerical choice of parameter values, in all cases these are chosen to ensure a frequency response 'backbone curve' is representative of a strongly nonlinear system with amplitudes an order of magnitude greater than 1 . The algorithm control parameters are supplied after the results. 


\subsection{Duffing model with single harmonic forcing}

In this first example the equation of motion is the familiar Duffing oscillator model:

$$
\ddot{y}+2 \xi \omega_{n} \dot{y}+\omega_{n}^{2} y+\varepsilon y^{3}=f(t)
$$

chosen with stiffness parameter values $\omega_{n}=1$ and $\varepsilon=0.75$, and damping level $\xi=0.05$, with single harmonic forcing $f(t)=\cos \left(\Omega_{1} t\right)$. Four iterations are made following the introduction of each additional low-frequency harmonic, so the maximum number of iterations per additional low-frequency harmonic is set to: $I_{\max }=4$. The maximum number of harmonics in the low-frequency group is set to $K_{\max }=8$. The 'switch number' is set to $K_{\text {switch }}=4$. This means in the iteration process, if the number of the harmonics in the low-frequency group $\leq 4$, equation (17) is used (which includes the SF-HBM combined with the arc-length term). But if the number of the low-frequency harmonics $>4$, equation (9) is used (i.e. with the arclength term deleted). The optimization interval is divided into 8 subintervals expressed as equation (22). The pre-set equation error criterion in Step 7 is set to $10^{-10}$.

Figures 3 - 7 show the results obtained using the proposed algorithm over $2^{6}$ discrete time points. Figure 3 shows the frequency response function over the entire frequency range with single harmonic forcing. The equation error RMS values for the first section of response function (i.e. with highest magnitude) are shown in figure 4, along with the equation error shown as a function of the total number of iterations. The error for the last iteration prior to the introduction of each additional low-frequency harmonic is shown with a 'o' symbol. In the response function curve, there are two turning points with frequency ratios $\Omega_{1} / \omega_{n}=2.8404$ and $\Omega_{1} / \omega_{n}=1.5975$. The solution information at the turning point frequency ratio $\Omega_{1} / \omega_{n}$ $=2.8404$ is shown in figures 5 to 7 . Figure 5 shows the absolute magnitudes of all 32 harmonics for the turning point frequency ratio 2.8404 . The corresponding equation error at the frequency ratio 2.8404 , as a function of the total number of iterations, is shown in figure 6. Figure 7 shows the solution obtained compared with the starting low-frequency harmonic, 
for which the mean is assumed to be zero and the magnitude, phase, and fundamental

frequency are starting guesses using equation (24).

The previous solution is the starting point for the current arc in the continuation process, so the computational solution accuracy of each step has heavy influence on the following traced solutions. The high solution accuracy in the second stage (with total iteration numbers 17 to 32 shown in figure 4) contributes to very favorable continuation accuracy, which is especially important when tracing solutions around a turning point. It can be seen that the equation error RMS value reduces rapidly in the second stage. Figure 4 shows that the equation error RMS value is very small. In summary, the high computational accuracy lays the foundation for efficient and effective tracing.

\subsection{An oscillator with non-expansible stiffness and with single harmonic forcing}

A test oscillator with a $9^{\text {th }}$ degree nonlinearity is shown in the form:

$$
\ddot{y}+2 \xi \omega_{n} \dot{y}+\omega_{n}^{2} y+\varepsilon y^{9}=f(t)
$$

with stiffness parameter values $\omega_{n}=1$ and $\varepsilon=0.2$, damping factor $\xi=0.04$, and with single harmonic forcing $f(t)=\cos \left(\Omega_{1} t\right)$. A high number of low-frequency harmonics is needed for convergence owing to the $9^{\text {th }}$ power nonlinearity. Therefore, the maximum number of harmonics in the low-frequency group is set to $K_{\max }=18$. The maximum number of iterations per additional low-frequency harmonic is set to $I_{\max }=4$. The 'switch number' is set to $K_{\text {switch }}=4$. In the iteration process, equation (16) is used if the number of the harmonics in the low-frequency group $\leq 4$; and equation (9) is used if the number of the low-frequency harmonics $>4$. The optimization interval is divided into 6 subintervals expressed by equation (22). The pre-set equation error criterion in Step 7 is set to $10^{-8}$.

Figures 8 - 11 show the results computed using the proposed algorithm over $2^{8}$ discrete time points. Figure 8 shows the corresponding frequency response function over the entire frequency range. The equation error RMS values for the first response function section (with 
highest magnitude) are shown in figure 9 . Two turning points are at frequency ratios $\Omega_{1} / \omega_{n}$

$=6.0090$ and $\Omega_{1} / \omega_{n}=1.4465$. Figure 10 shows the absolute magnitudes of the Fourier coefficients for the total 128 solution harmonics at the turning point frequency ratio $\Omega_{1} / \omega_{n}$ $=6.0090$. Figure 11 gives the equation error RMS values as a function of the total number of iterations.

\subsection{An oscillator with non-expansible stiffness and with 24-harmonic forcing}

The test oscillator described by equation (27) with the same previous model parameters is used again but now with 24-harmonic periodic forcing expressed as:

$$
\begin{aligned}
f(t)= & 1.1908 \cos 2 \Omega_{1} t+0.0000 \sin 2 \Omega_{1} t-1.2025 \cos 4 \Omega_{1} t-0.3179 \sin 4 \Omega_{1} t \\
& -0.0198 \cos 6 \Omega_{1} t+1.0950 \sin 6 \Omega_{1} t-0.1567 \cos 8 \Omega_{1} t-1.8740 \sin 8 \Omega_{1} t \\
& -1.6041 \cos 10 \Omega_{1} t+0.4282 \sin 10 \Omega_{1} t+0.2573 \cos 12 \Omega_{1} t+0.8956 \sin 12 \Omega_{1} t \\
& -1.0565 \cos 14 \Omega_{1} t+0.7310 \sin 14 \Omega_{1} t+1.4151 \cos 16 \Omega_{1} t+0.5779 \sin 16 \Omega_{1} t \\
& -0.8051 \cos 18 \Omega_{1} t+0.0403 \sin 18 \Omega_{1} t+0.5287 \cos 20 \Omega_{1} t+0.6771 \sin 20 \Omega_{1} t \\
& +0.2193 \cos 22 \Omega_{1} t+0.5689 \sin 22 \Omega_{1} t-0.9219 \cos 24 \Omega_{1} t-0.2556 \sin 24 \Omega_{1} t \\
& -2.1707 \cos 26 \Omega_{1} t-0.3775 \sin 26 \Omega_{1} t-0.0592 \cos 28 \Omega_{1} t-0.2959 \sin 28 \Omega_{1} t \\
& -1.0106 \cos 30 \Omega_{1} t-1.4751 \sin 30 \Omega_{1} t+0.6145 \cos 32 \Omega_{1} t-0.2340 \sin 32 \Omega_{1} t \\
& +0.5077 \cos 34 \Omega_{1} t+0.1184 \sin 34 \Omega_{1} t+1.6924 \cos 36 \Omega_{1} t+0.3148 \sin 36 \Omega_{1} t \\
& +0.5913 \cos 38 \Omega_{1} t+1.4435 \sin 38 \Omega_{1} t-0.6436 \cos 40 \Omega_{1} t-0.3510 \sin 40 \Omega_{1} t \\
& +0.3803 \cos 42 \Omega_{1} t+0.6232 \sin 42 \Omega_{1} t-1.0091 \cos 44 \Omega_{1} t+0.7990 \sin 44 \Omega_{1} t \\
& -0.0195 \cos 46 \Omega_{1} t+0.9409 \sin 46 \Omega_{1} t-0.0482 \cos 48 \Omega_{1} t-0.9921 \sin 48 \Omega_{1} t
\end{aligned}
$$

The harmonic coefficients for the 24-harmonic forcing, shown in Equation (28), were drawn randomly from a normal distribution $\mathrm{N}(0,1)$ each with the same fundamental period as the single harmonic case. The coefficients are given to an accuracy of four decimal places. The excitation obviously includes many more harmonics, so the maximum number of harmonics for the low-frequency group has to be increased for convergence and set to $K_{\max }=20$. The computational parameters are re-used as in previous single harmonic forcing test.

Figures $12-15$ show, for 24-harmonic forcing, the results obtained using the proposed 7- 
step algorithm. Figure 12 shows the frequency response function spanning the frequency

range from the turning point frequency ratio $\Omega_{1} / \omega_{n}=1.5269$ to a sufficiently high value $\Omega_{1} / \omega_{1}=8$. The equation error RMS values for the first response section (i.e. with low magnitude) are shown in Figure 13. Figure 14 and 15 give the absolute magnitudes of the total 128 response harmonics, and equation error RMS at the turning point frequency ratio $\Omega_{1} / \omega_{n}=1.5269$.

\section{The Algorithm Control Parameters}

Before discussing the results associated with the three previous numerical examples the algorithm control parameters are now given. First concerning the arc-length factor $\lambda$, in Step 1 of the 7-step algorithm of Section 4 (combining the SF-HBM and ALC), this is initially set to the value $\lambda=1$. Later, if the equation error is unacceptably large, $\lambda$ is successively reduced in size in Step 7 as shown in the flowchart in Figure 2. If, for small $\lambda$ (i.e. typically two orders of magnitude smaller than the initial value) the equation error is still too large, this indicates a turning point. The calculation is then stopped, and the arc-length factor is reset to the value $\lambda=1$. During the iteration, the magnitude of $\lambda$ is implicitly set during iteration by satisfying the equation $s_{s e t}=\lambda s_{0}$ where $s_{s e t}$ is the 'pre-set' arc length. The initial arc-length $s_{0}$ is obtained at the two starting points (i.e. as shown in figure 2, computed by the SF-HBM at the two starting frequencies) and is therefore a variable. The (pre-set limit) arc-length factor $\lambda$ is set by specifying $s_{\text {set }}$ for the iteration. The accuracy of the predicted frequency at a turning point depends on this limit. The pre-set arc length $s_{\text {set }}$ is selected largely from experience. For example, in the Duffing model example in Section 5.1, the chosen value was $s_{\text {set }}=0.05$. For the oscillator example in Section 5.2, with the 9th degree nonlinear stiffness and single harmonic forcing, the chosen value was $s_{\text {set }}=10^{-6}$ because the shape of the turning point is extremely sharp. Whereas for the oscillator example in Section 5.3 with the 9th degree nonlinear stiffness and multi-harmonic forcing, the chosen 
value was $s_{\text {set }}=0.005$ because the turning point was less sharp.

The pre-set equation error criterion in Step 7 is problem specific - in the first example (i.e. the Duffing model) this was set to $10^{-10}$ whereas in the second and third examples it was set to $10^{-8}$. The reason for this difference is that the second and third examples have $9^{\text {th }}$ degree nonlinearity (rather than cubic) making it more difficult to find a solution. A less stringent equation error criterion of $10^{-8}$ guarantees higher chances of success in finding a solution. However, it can be seen that the RMS equation error is actually lower than $10^{-10}$ in all of the examples.

\section{Discussion of Results}

There is a noticeable difference between the frequency response function for Section 5.3 compared with those shown in figures 7 and 8 . The response function does not exist in the latter case at a frequency ratio below the turning point frequency ratio $\Omega_{1} / \omega_{n}=1.5269$. In other words, based on the pre-set equation error criterion of $10^{-8}$ in Step 7, the corresponding solution cannot be obtained using the proposed algorithm. This can be explained using the results shown in figure 16, which are obtained using numerical simulation via Matlab. To get a very accurate solution by simulation, the simulation time has to be sufficiently long, for example from 0 to 3000 seconds. Figure 16 shows the excitation forces at the specific frequency (ratio) $\pi / 2=1.5707$, and two sets of simulated time-domain responses at two specific frequency ratios $(\pi / 2=1.5707$ and 1.5270$)$ which are above the turning point frequency ratio 1.5269 , plus three additional sets of simulated time-domain responses, at three specific frequency ratios $(\pi / 6=0.5236, \pi / 3=1.0472$ and 1.5268$)$, which are below the turning point frequency ratio 1.5269 . It can be seen that the solutions at the frequency ratio lower than 1.5269 , are not periodic, so the basic prerequisite for the HMB is not satisfied, namely that the oscillator expressed by equation (1) and has a periodic response. If the frequency ratio is greater than 1.5269 , the system response is periodic, and the 
proposed algorithm can compute frequency response function information with high

accuracy. In addition, the traced frequency response function comprises two segments: a high magnitude segment and a low magnitude segment, as shown in figure 12. The solutions on the low magnitude segment are stable and can be obtained using simulation, two of which are at two specific frequency ratios $(\pi / 2=1.5707$ and 1.5270$)$ shown in figure 16 , while the solutions on the high magnitude segment are unstable.

The fidelity of the predicted turning point frequency ratio 1.5269 is clearly verified using the time-domain simulation results. By using the predicted turning point frequency ratio of 1.5269 and increasing it to 1.5270 (i.e. by 0.0001 ) the simulation model gives a stable and periodic solution as shown in figure 16. But the solution given by simulation at the frequency ratio 1.5268 (a very small percentage less than the turning point frequency of 1.5269 ) is not periodic, as shown in figure 16. A turning point is the transition between stable and unstable solutions (shown in figures 8 and 12, for example). The optimal frequency in the arc-length continuation process is restricted to the optimal interval shown in equation (22). This is controlled by the pre-set arc-length as shown in equation (21). The arc-length is controlled in Step 7 by the pre-set limit arc-length factor $\lambda$. The frequency accuracy can therefore easily be controlled. The arc-length is actually controlled to be not less than 0.005 in the third numerical example. In other words, the current solution is regarded as a turning point if the current arc-length is less than 0.005 . However, it can be seen that the accuracy of the frequency ratio at the turning point is very good, where the frequency ratio error is smaller than 0.0002 as shown in figure 16 . Failure of the iteration process to converge with high computational accuracy (for example, $10^{-10}$ in the first example, and $10^{-8}$ in the second and third examples) may infer either that a Period-1 response does not exist i.e. the response does not have the same fundamental period as the excitation, in which case sub-harmonic responses should be considered. Or it may indicate that there is a totally non-periodic response in the form of chaos, which does not satisfy the basic prerequisite for the HMB, 
and therefore not surprisingly the method fails to converge. Overall for high accuracy, the

proposed 7-step algorithm can provide fast frequency response information for oscillators with high degree nonlinearities.

\section{CONCLUSIONS}

Accurate frequency analysis of oscillators with non-expansible, high degree polynomial nonlinearities and multi-harmonic forcing, has been addressed to overcome the low computational accuracy and efficiency faced by the HBM when tracing around turning points using ALC. A new 7-step algorithm has been proposed by combining the SF-HBM with arclength continuation in a two-stage scheme. Stage I guarantees the entire response can be traced with high accuracy and efficiency. Stage II exploits the excellent computational efficiency and accuracy of the SF-HBM. Test results obtained for Period-1 responses in three numerical examples show that the proposed algorithm offers three marked advantages over conventionally-combined HBM and ALC. The first advantage is its accuracy and efficiency in tracing all solutions. The second advantage is that a turning-point frequency can be traced to any required accuracy. And the third advantage is that it allows oscillators with nonexpansible nonlinearities and multiple harmonic forcing to be handled without difficulty because the system of algebraic HBM equations can be easily generated using the FFT.

\section{ACKNOWLEDGEMENTS}

The authors acknowledge with sincere gratitude, the National Science Foundation of China (No. U1304525), and the Scholarship Fund of China Scholarship Council (File No.201408410031), for providing support for this research collaboration. 


\section{References:}

[1] Z. F. Xin, S. A. Neild, D. J. Wagg, Z.X. Zuo, Resonant response functions for nonlinear oscillators with polynomial type nonlinearities, Journal of Sound and Vibration, 332 (2013) 1777-1788.

[2] A. Sofi, Nonlinear in-plane vibrations of inclined cables carrying moving oscillators, Journal of Sound and Vibration, 332 (2013) 1712-1724.

[3] O.V. Gendelman, T. Sapsis, A.F. Vakakis, L.A. Bergman, Enhanced passive targeted energy transfer in strongly nonlinear mechanical oscillators, Journal of Sound and Vibration, 330 (2011) 18.

[4] C. Sun, R.P. Eason, S. Nagarajaiah, A.J. Dick,Hardening, Duffing oscillator attenuation using a nonlinear TMD, a semi-active TMD and multiple TMD, Journal of Sound and Vibration, 332 (2013) 674-686.

[5] J.S.A.E. Fouda, B. Bodo, G.M.D. Djeufa, S.L. Sabat, Experimental chaos detection in the Duffing oscillator, Communications in Nonlinear Science and Numerical Simulation, 33 (2016) 259-269.

[6] J.C. Ji, Two families of super-harmonic resonances in a time-delayed nonlinear oscillator, Journal of Sound and Vibration, 349 (2015) 299-314.

[7] O.V. Pischanskyy, W.T. Van Horssen, On the nonlinear dynamics of a single degree of freedom oscillator with a time-varying mass, Journal of Sound and Vibration, 331 (2012) 1887-1897.

[8] A.E. S. Leadenham, M-shaped asymmetric nonlinear oscillator for broadband vibration energy harvesting: Harmonic balance analysis and experimental, Journal of Sound and Vibration, 333 (2014) 6209-6223.

[9] G. Gatti, M.J. Brennan, On the effects of system parameters on the response of a harmonically excited system consisting of weakly coupled nonlinear and linear oscillators, Journal of Sound and Vibration, 330 (2011) 4538-4550.

[10] G. Gatti, I. Kovacic, M.J. Brennan, On the response of a harmonically excited two degree-offreedom system consisting of a linear and a nonlinear quasi-zero stiffness oscillator, Journal of Sound and Vibration, 329 (2010) 1823-1835.

[11] Y. Starosvetsky, O.V. Gendelman, Response regimes of linear oscillator coupled to nonlinear energy sink with harmonic forcing and frequency detuning, Journal of Sound and Vibration, 15 (2008) 746-765.

[12] J.I. Ramos, An artificial parameter Linstedt-Poincaré method for the periodic solutions of nonlinear oscillators in which the restoring force is inversely proportional to the dependent variable, Journal of Sound and Vibration,318(2008)1281-1290.

[13] Albert C. J. Luo, Jr. P. Zwiegart, Existence and analytical predictions of periodic motions in a periodically forced, nonlinear friction oscillator, Journal of Sound and Vibration, 309 (2008) 129-149.

[14] G. Von Groll, D.J. Ewins, The harmonic balance method with arc-length continuation in rotor/stator contact problems, Journal of Sound and Vibration, 241 (2001) 223-233.

[15] J.F. Dunne, P. Hayward, A split-frequency harmonic balance method for nonlinear oscillators with multi-harmonic forcing, Journal of Sound and Vibration, 295 (2006) 939-963.

[16] J.F. Dunne, Subharmonic-Response Computation and Stability Analysis for a Nonlinear Oscillator Using a Split-Frequency Harmonic Balance Method, Journal of Computational and Nonlinear Dynamics, 1, (2006) 221-229. 
[17] H. Hu, Solution of a mixed parity nonlinear oscillator: Harmonic balance, Journal of Sound and Vibration 299 (2007) 331-338.

[18] H. Hu, Exact solution of a quadratic nonlinear oscillator, Journal of Sound and Vibration, 295 (2006) 450-457.

[19] G. Zakeri, E. Yomba, Exact solutions of a generalized autonomous Duffing-type equation, Applied Mathematical Modeling, 39 (2015) 4607-4616.

[20] M.C. Depassier, V. Haikala, Analytic upper and lower bounds for the period of nonlinear oscillators, Journal of Sound and Vibration, 328 (2009) 338-344.

[21] I.V. Andrianov, W.T. van Horssen, Analytical approximations of the period of a generalized nonlinear van der Pol oscillator, Journal of Sound and Vibration, 295 (2006) 1099-1104.

[22] A.H. Nayfeh, D.T. Mook, Nonlinear Oscillations, Wiley Classics Library Edition,1995.

[23] I. Kovacic, Forced vibrations of oscillators with a purely nonlinear power-form restoring force, Journal of Sound and Vibration, 330 (2011) 4313-4327.

[24] A.H.N.P. Malatkar, Calculation of the jump frequencies in the response of s.d.o.f. non-linear systems, Journal of Sound and Vibration, 254 (2002) 1005-1011.

[25] A. Beléndez, D.I. Méndez, T. Beléndez, A. Hernández, M.L. Álvarez, Harmonic balance approaches to the nonlinear oscillators in which the restoring force is inversely proportional to the dependent variable, Journal of Sound and Vibration 314(3) (2008) 775-782.

[26] A. Beléndez, A. Hernández, T. Beléndez, M.L. Álvarez, S. Gallego, M. Ortuño, C. Neipp, Application of the harmonic balance method to a nonlinear oscillator typified by a mass attached to a stretched wire, Journal of Sound and Vibration, 302 (2007) 1018-1029.

[27] B. Zhou, F. Thouverez, D. Lenoir, A variable-coefficient harmonic balance method for the prediction of quasi-periodic response in nonlinear systems, Mechanical Systems and Signal Processing, 64-65 (2015) 233-244.

[28] A. Beléndez, E. Gimeno, M.L. Alvarez, D.I. Méndez, Nonlinear oscillator with discontinuity by generalized harmonic balance method, Computers \&Mathematics with Applications, 58 (2009) 21172123.

[29] T. Detroux, L. Renson, L. Masset, G. Kerschen, The harmonic balance method for bifurcation analysis of large-scale nonlinear mechanical systems, Computers \& Mathematics with Applications, 296 (2015) 18-38.

[30] D W Luerkens, Theory and applications of morphological analysis - fine particles and surfaces, Appendix II pp 271-274. CRC press (1991) ISBN 9780849367779.

[31] K.B. Blair, C.M. Krousgrill, T.N. Farris, Harmonic balance and continuation techniques in the dynamic analysis of duffing's equation, Journal of Sound and Vibration, 202 (1997) 717-731.

[32] S. Karkar, B. Cochelin, C. Vergez, A high-order, purely frequency based harmonic balance formulation for continuation of periodic solutions: The case of non-polynomial nonlinearities, Journal of Sound and Vibration, 332 (2013) 968-977.

[33] C. Piccardi, Bifurcations of limit cycles in periodically forced nonlinear systems: The harmonic balance approach, IEEE Trans. Circuits Syst. I, 41 (4) (1994), pp. 315-320. 
[34] R.E. Mickens, A generalized iteration procedure for calculating approximations to periodic solutions of "truly nonlinear oscillators", Journal of Sound and Vibration, 287 (2005) 1045-1051.

[35] A. Grolet, F. Thouverez, On a new harmonic selection technique for harmonic balance method, Mech. Syst. Signal Process., 30 (2012), pp. 43-60.

[36] A.Y.T. Leung, Z. Guo and T.C. Fung. The multi-parameter homotopy harmonic balance method for steady state problems, International Journal of Computer Mathematics, 87(5):1158-1177, March 2010.

[37] $\mathrm{H}$. Chen and $\mathrm{M}$. Ni, Iterative Homotopy Harmonic Balance Approach for Determining the Periodic Solution of a Strongly Nonlinear Oscillator, Mathematical Problems in Engineering, Volume 2015 (2015).

[38] R. Viguié, G. Kerschen. Design Procedure of a Nonlinear Vibration Absorber Using Bifurcation Analysis, 2009 IMAC-XXVII: Conference \& Exposition on Structural Dynamics (12 pages). 


\section{LIST OF FIGURES}

Figure 1. Tracing process of the SF-HBM in conjunction with arc-length continuation.

Figure 2. Flowchart for the proposed 7-step algorithm.

Figure 3. Frequency response function for Duffing oscillator with single harmonic forcing.

Figure 4. Equation error RMS as a function of iteration number with four iterations as each of the eight lowfrequency harmonics is introduced $\left(4^{\text {th }}\right.$ iteration shown overlaid with O symbol)) for Duffing oscillator with single harmonic forcing.

Figure 5. Absolute magnitudes of the total 32 response harmonics at turning point frequency ratio $\Omega_{1} / \omega_{n}=2.8404$ for Duffing oscillator with single harmonic forcing.

Figure 6. Equation error RMS at turning point frequency ratio $\Omega_{1} / \omega_{n}=2.8404$ for Duffing oscillator with single harmonic forcing.

Figure 7. Periodic solution compared with starting single harmonic at turning point frequency ratio $\Omega_{1} / \omega_{n}=2.8404$ for Duffing oscillator with single harmonic forcing.

Figure 8. Frequency response function for oscillator with non-expansible stiffness with single harmonic forcing.

Figure 9. Equation error RMS as a function of iteration number with four iterations as each of the eight lowfrequency harmonics is introduced ( $4^{\text {th }}$ iteration shown overlaid with $\mathrm{O}$ symbol)) for oscillator with nonexpansible stiffness with single harmonic forcing.

Figure 10. Absolute magnitudes of the total 128 response harmonics at turning point frequency ratio $\Omega_{1} / \omega_{n}=6.0090$ for oscillator with non-expansible stiffness with single harmonic forcing.

Figure 11. Equation error RMS at turning point frequency ratio $\Omega_{1} / \omega_{n}=6.0090$ oscillator with nonexpansible stiffness with single harmonic forcing.

Figure 12. Frequency response function for oscillator with non-expansible stiffness and 24-harmonic forcing.

Figure 13. Equation error RMS as a function of iteration number with four iterations as each of the 20 lowfrequency harmonics is introduced ( $4^{\text {th }}$ iteration shown overlaid with $\mathrm{O}$ symbol)) for oscillator with nonexpansible stiffness and 24-harmonic forcing.

Figure 14. Absolute magnitudes of the total 128 response harmonics at turning point frequency ratio $\Omega_{1} / \omega_{n}=1.5269$ for oscillator with non-expansible stiffness and 24-harmonic forcing.

Figure 15. Equation error RMS at turning point frequency ratio $\Omega_{1} / \omega_{n}=1.5269$ for oscillator with nonexpansible stiffness and 24-harmonic forcing.

Figure 16. Periodic excitation and simulation response at specific frequency ratios. 


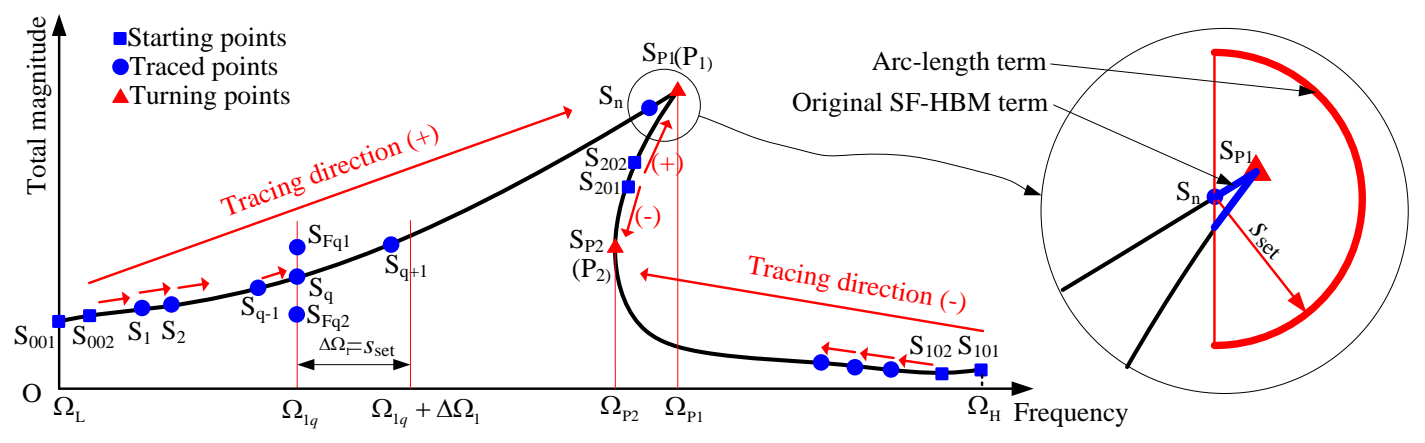

Figure1. Tracing process of the SF-HBM in conjunction with arc-length continuation. 
\begin{tabular}{|l|}
\hline $\begin{array}{r}\text { Step 1: } \\
\text { Select the parameters for outer loop } \\
\text { and select accurate starting values }\end{array}$ \\
\hline 1) Compute starting solutions $X_{-1}, X_{0}$ using SF-HBM. \\
2) Compute the arc-length $s_{0}$ using Eq. (14). \\
3) Set the arc-length factor $\lambda=1$. \\
4) Set q=1 (outer loop variable).
\end{tabular}

\section{Step 2: Select the parameters for the middle and inner loops}

1) Compute initial guesses using Eq. (24).

2) Set $y_{H F O}(t)=0$

3) Set $k=1$ (middle loop variable).

4) Set $i=1$ (inner loop variable).

\section{Step 3: Compute the amplitudes and phases associated with the low frequency group}

1) Set current arc-length $s_{\mathrm{set}}=\lambda s_{0}$

2) Express the solution as Eq. (25)

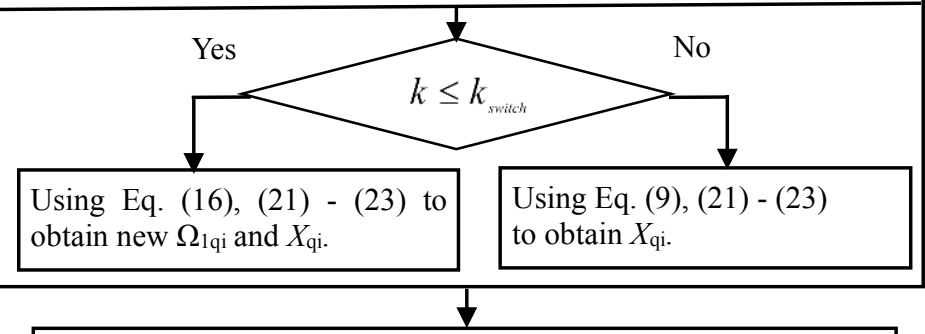

\section{Step 4: Compute the high frequency group}

1) Obtain the Fourier components of Eq. (18) using $\Omega_{1 q i .}$

2) Update the high-frequency group $y_{H F I}$ using Eq. (19).

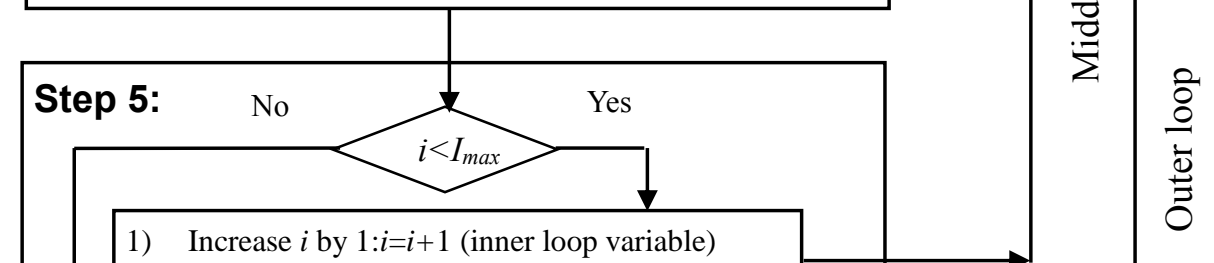

Increase $i$ by $1: i=i+1$ (inner loop variable)

2) Set $\Omega_{1 \mathrm{qi}}$ and $X_{\mathrm{qi}}$ as the initial guesses.

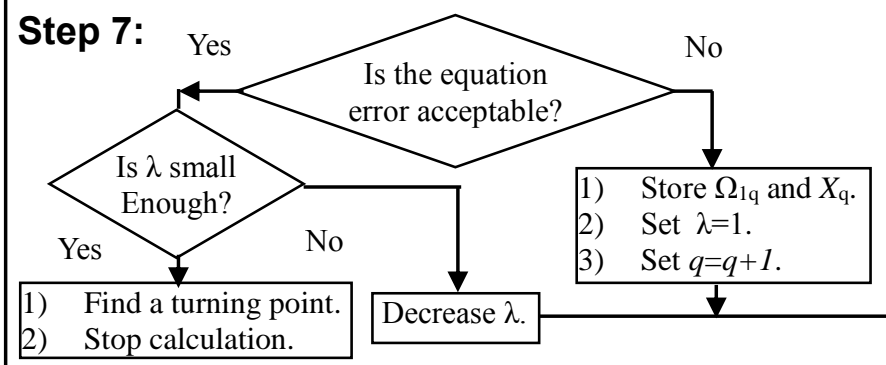




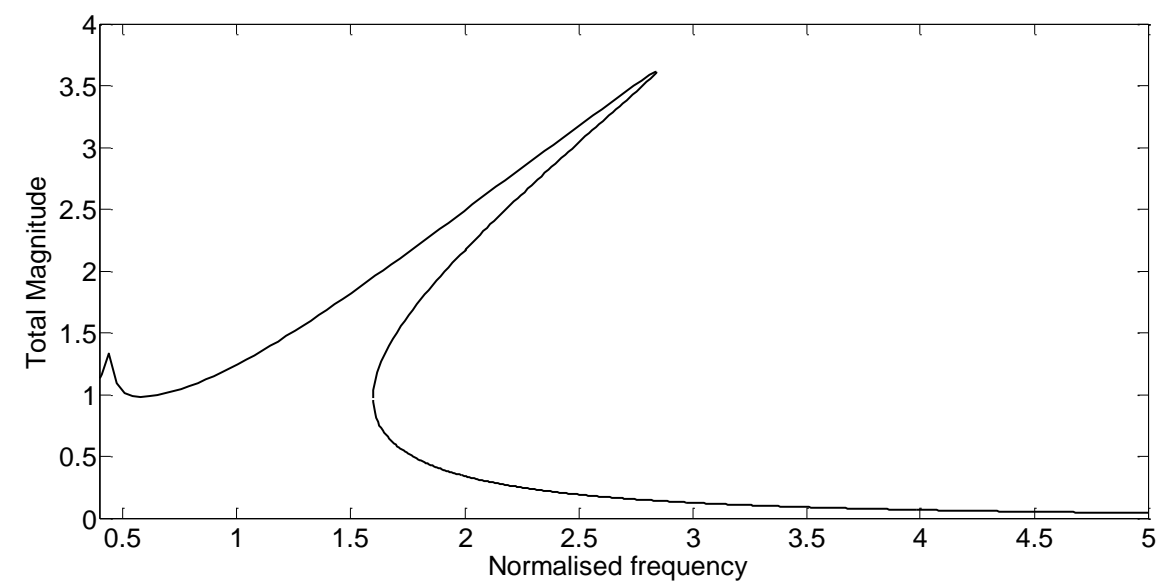

Figure 3. Frequency response function for Duffing oscillator with single harmonic forcing.

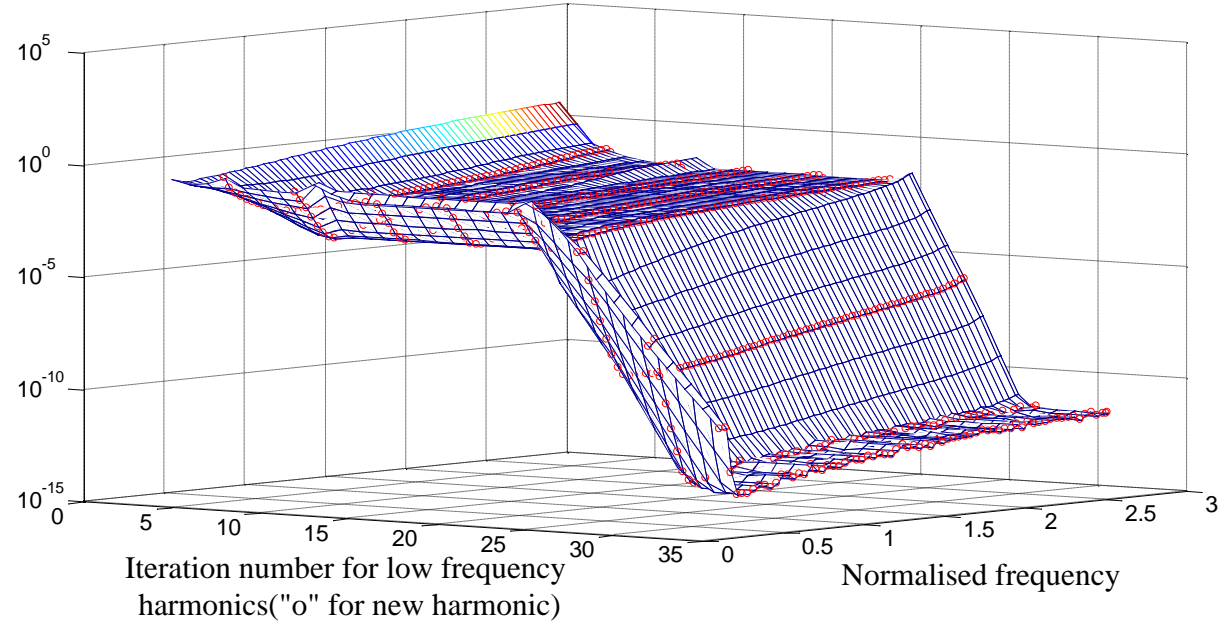

Figure 4. Equation error RMS as a function of iteration number with four iterations as each of the eight low-frequency harmonics is introduced $\left(4^{\text {th }}\right.$ iteration shown overlaid with 0 symbol)) for Duffing oscillator with single harmonic forcing.

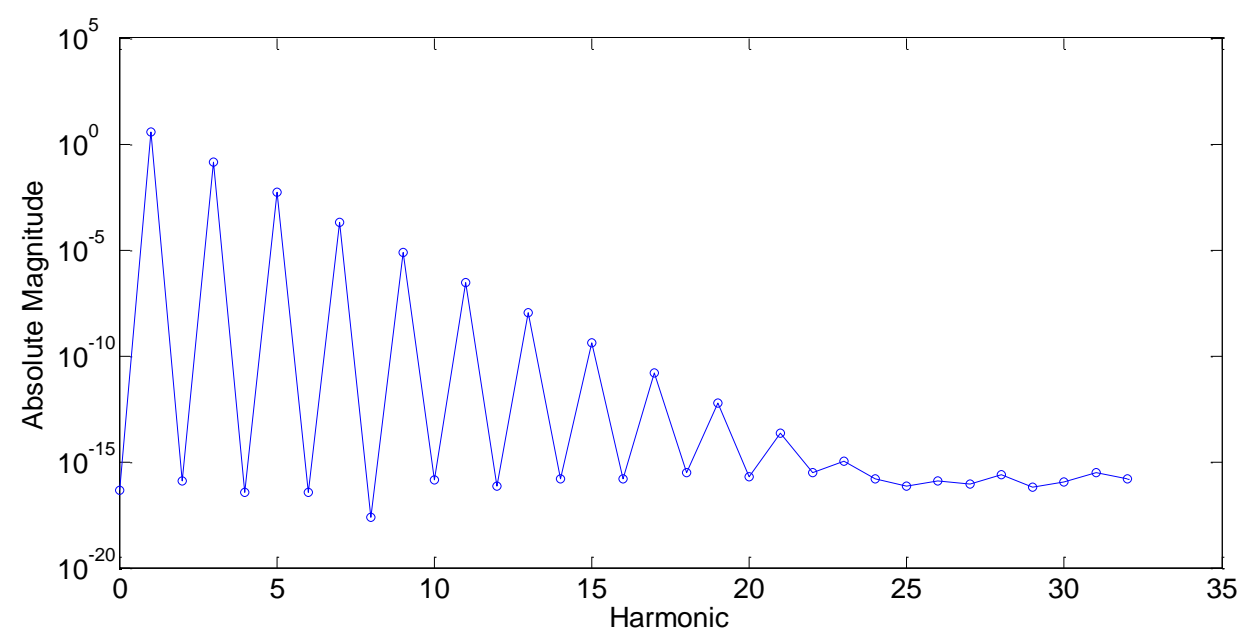

Figure 5. Absolute magnitudes of the total 32 response harmonics at turning point frequency ratio $\Omega_{1} / \omega_{n}=\mathbf{2 . 8 4 0 4}$ for Duffing oscillator with single harmonic forcing. 


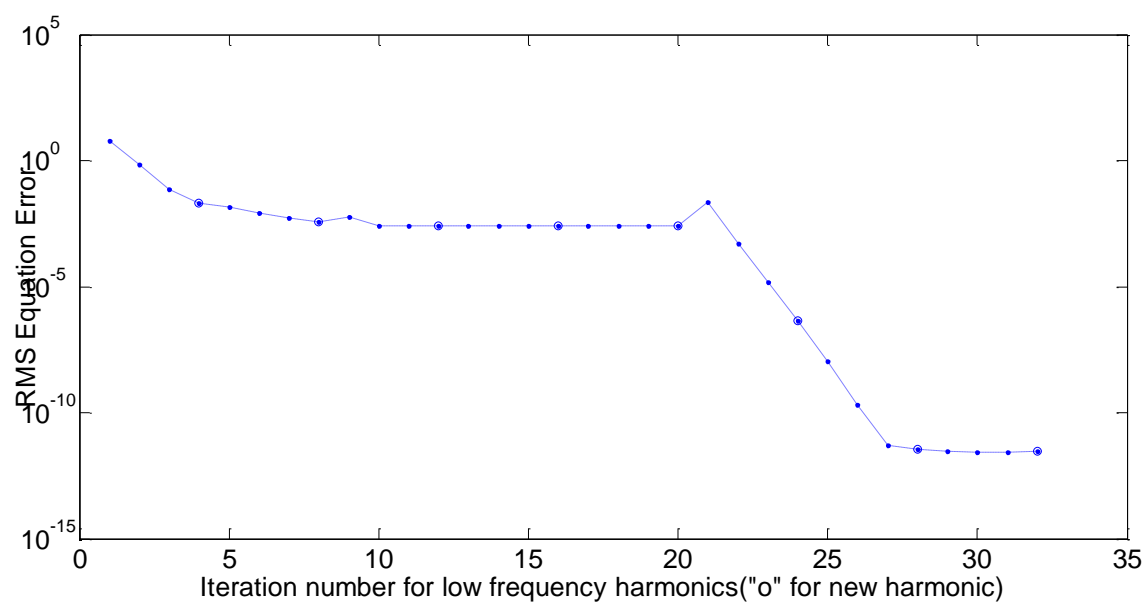

Figure 6. Equation error RMS at turning point frequency ratio $\Omega_{1} / \omega_{n}=\mathbf{2 . 8 4 0 4}$ for Duffing oscillator with single harmonic forcing.

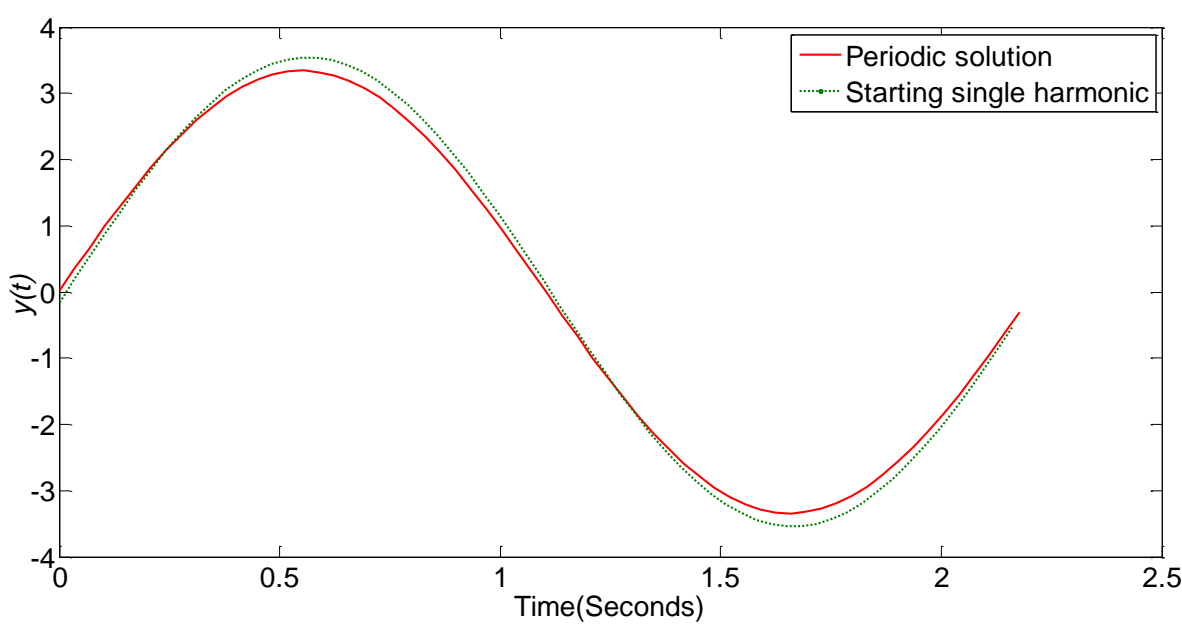

Figure 7. Periodic solution compared with starting single harmonic at turning point frequency ratio $\Omega_{1} / \omega_{n}=2.8404$ for Duffing oscillator with single harmonic forcing. 


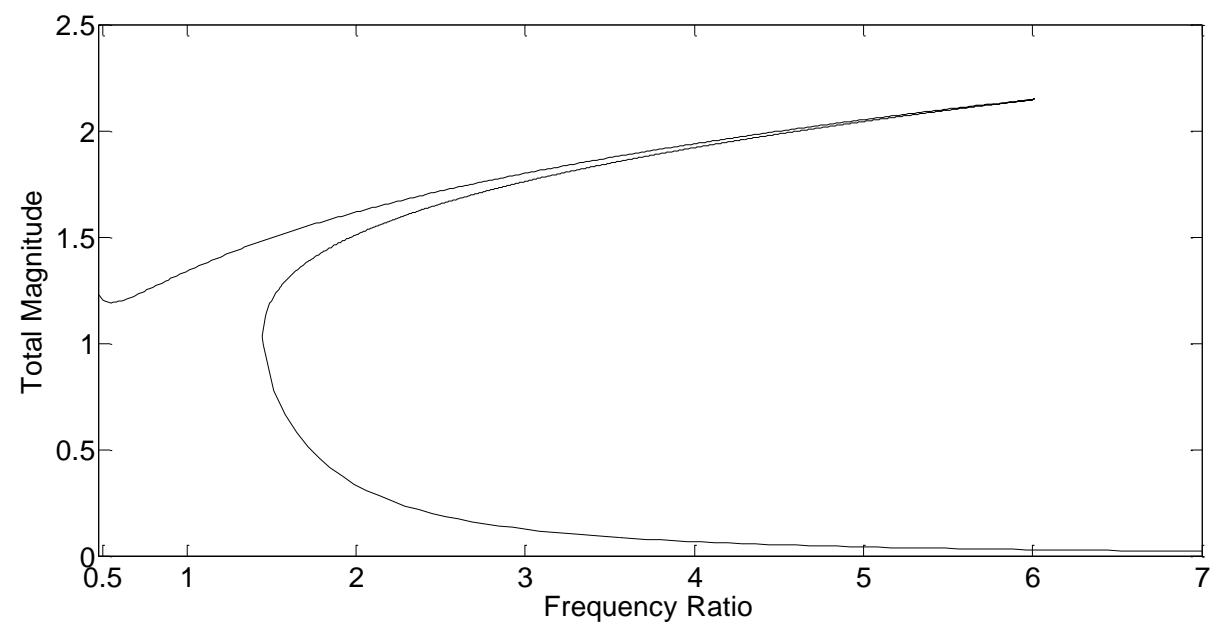

Figure 8. Frequency response function for oscillator with non-expansible stiffness with single harmonic forcing.

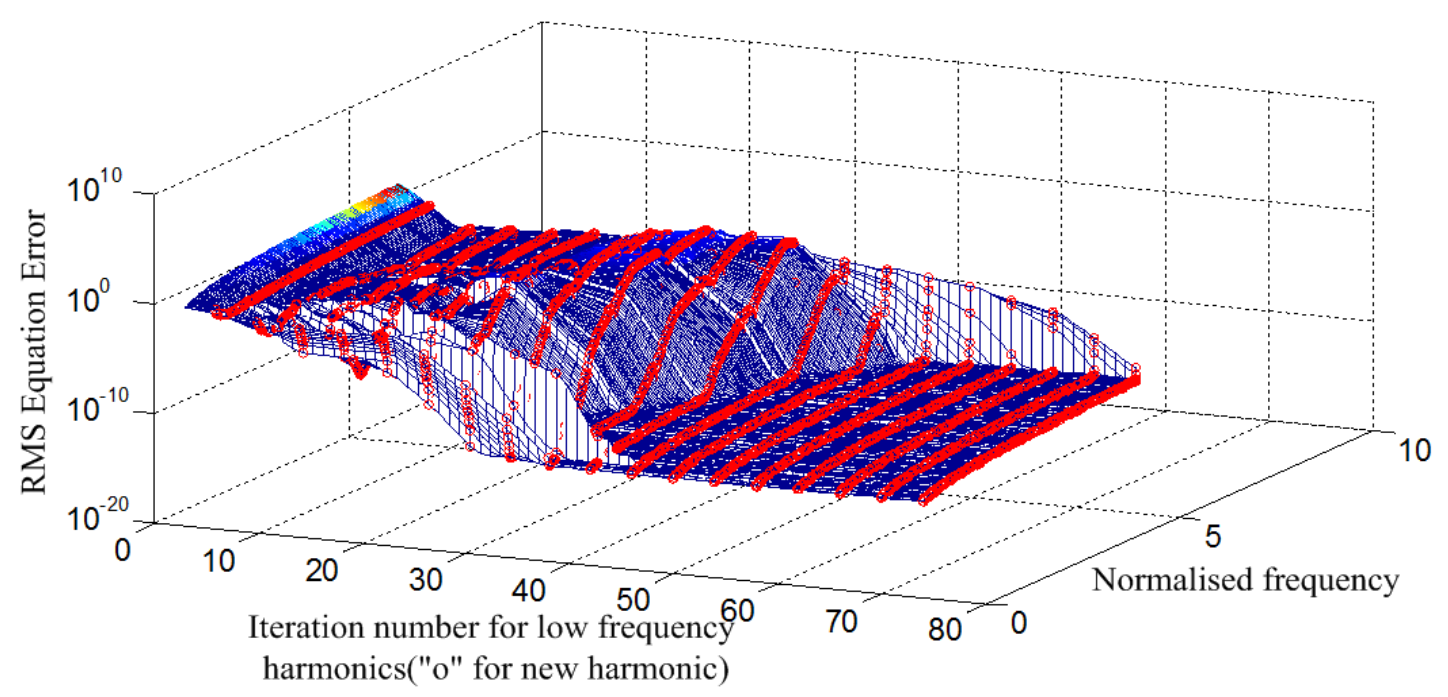

Figure 9. Equation error RMS as a function of iteration number with four iterations as each of the eight low-frequency harmonics is introduced $\left(4^{\text {th }}\right.$ iteration shown overlaid with $O$ symbol)) for oscillator with non-expansible stiffness with single harmonic forcing.

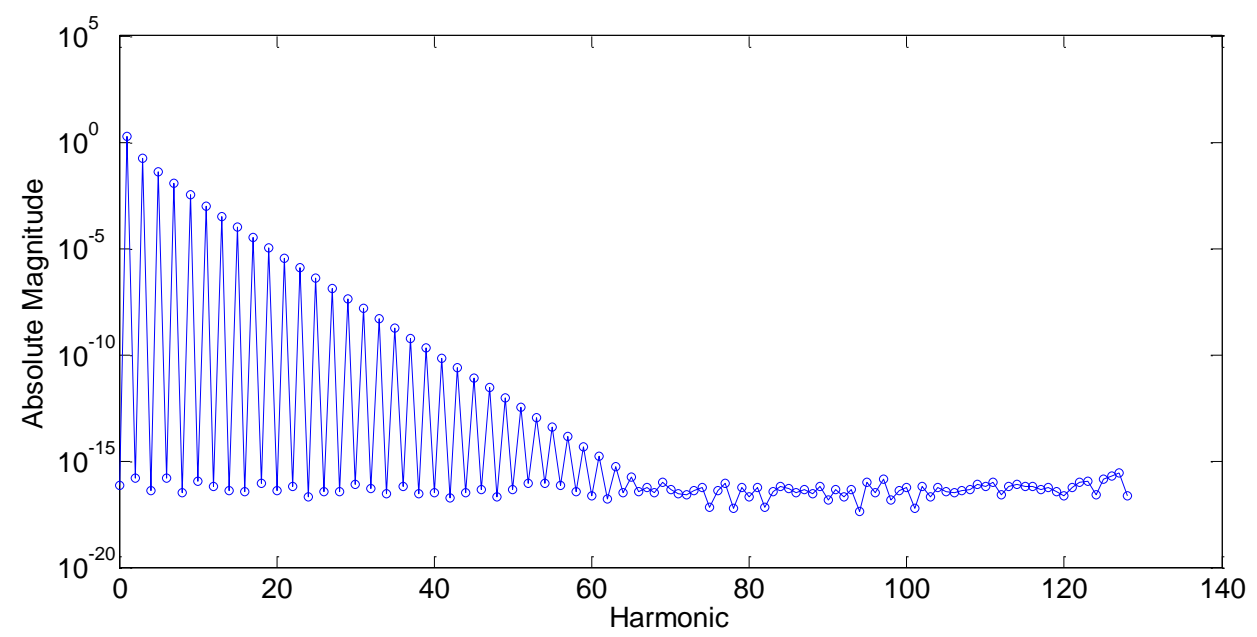

Figure 10. Absolute magnitudes of the total 128 response harmonics at turning point frequency ratio $\Omega_{1} / \omega_{n}=\mathbf{6 . 0 0 9 0}$ for oscillator with non-expansible stiffness with single harmonic forcing. 


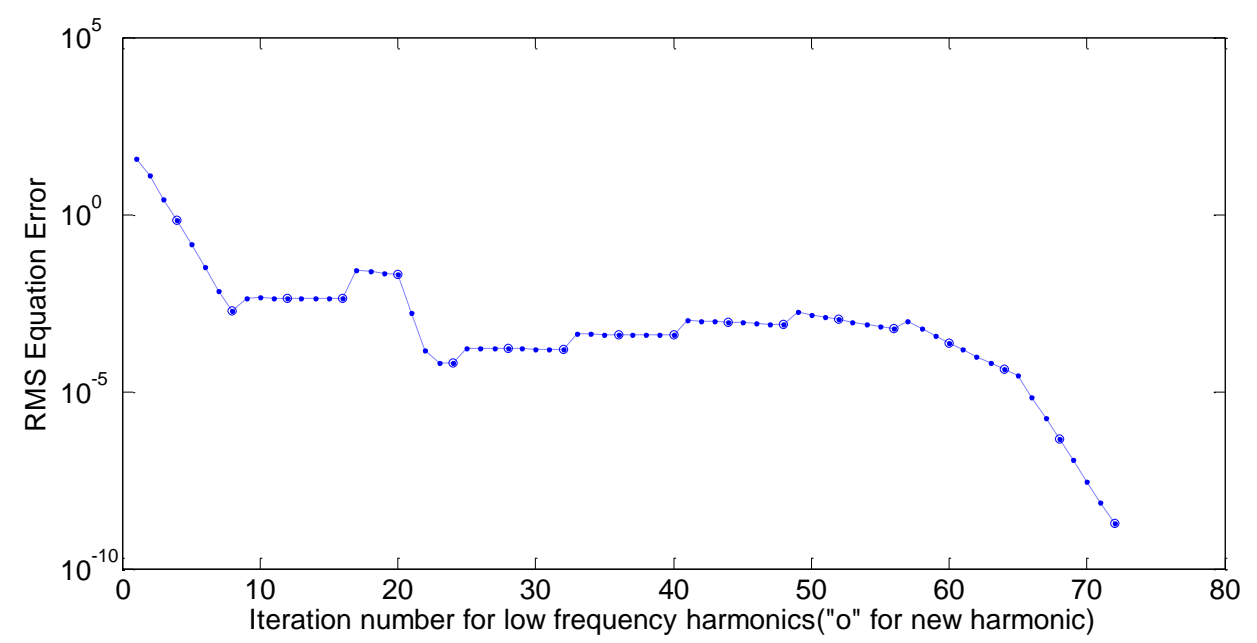

Figure 11. Equation error RMS at turning point frequency ratio $\Omega_{1} / \omega_{n}=\mathbf{6 . 0 0 9 0}$ oscillator with non-expansible stiffness with single harmonic forcing

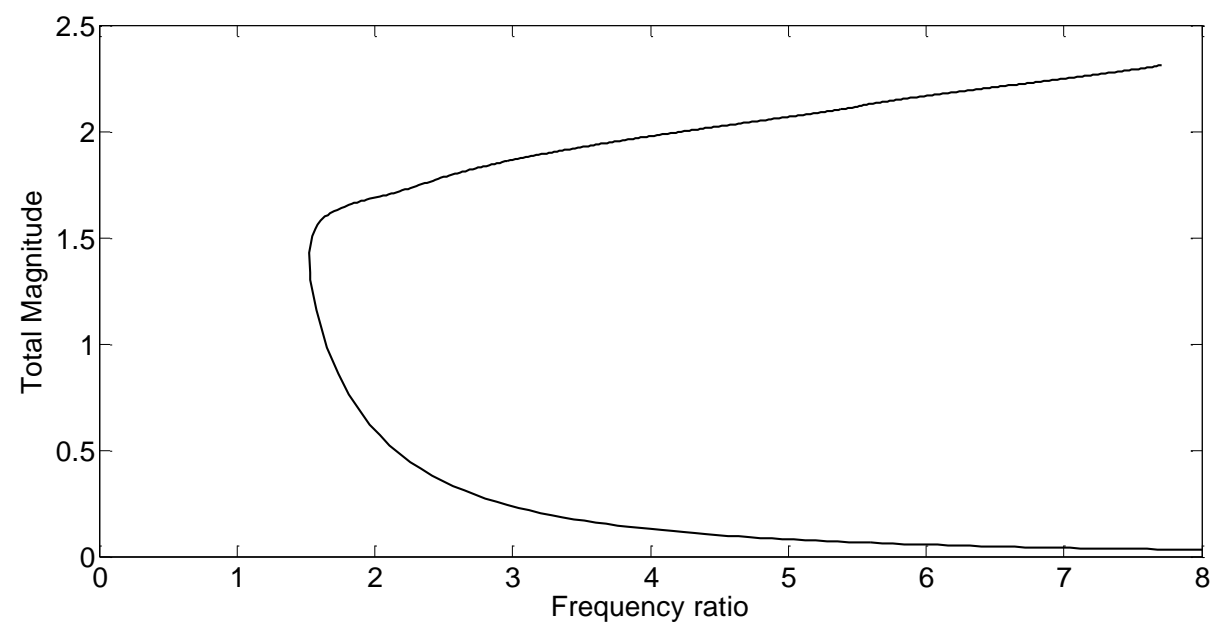

Figure 12. Frequency response function for oscillator with non-expansible stiffness and 24-harmonic forcing. 


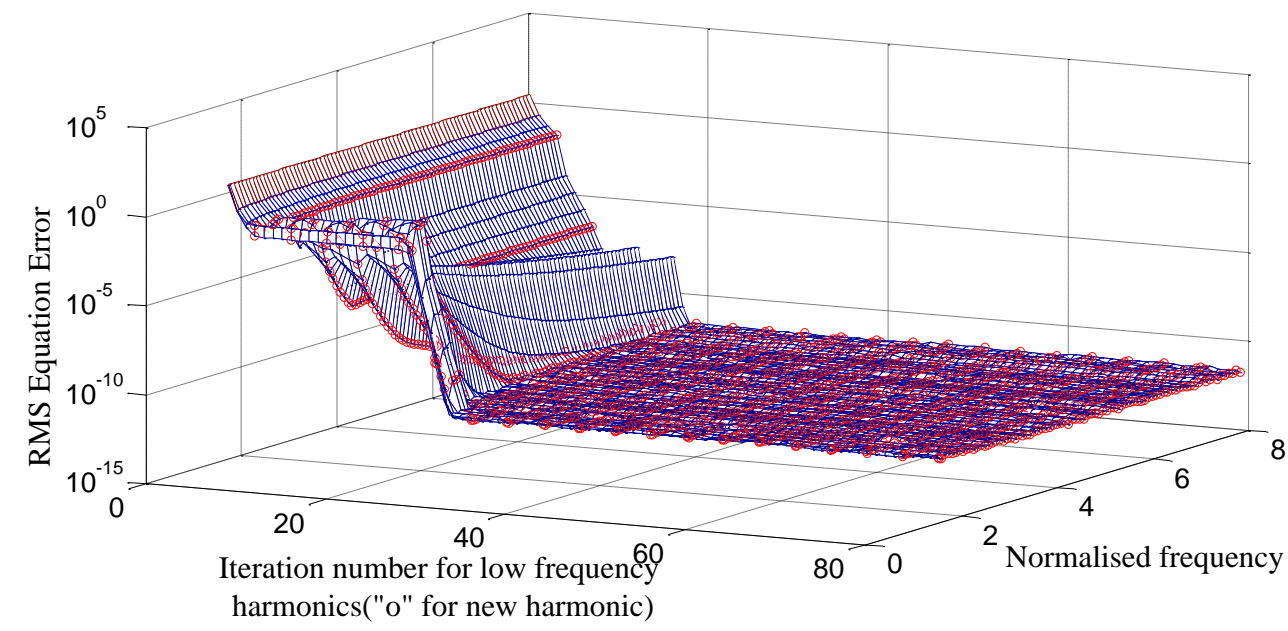

Figure 13. Equation error RMS as a function of iteration number with four iterations as each of the 20 low-frequency harmonics is introduced ( $4^{\text {th }}$ iteration shown overlaid with 0 symbol) for oscillator with non-expansible stiffness and 24-harmonic forcing.

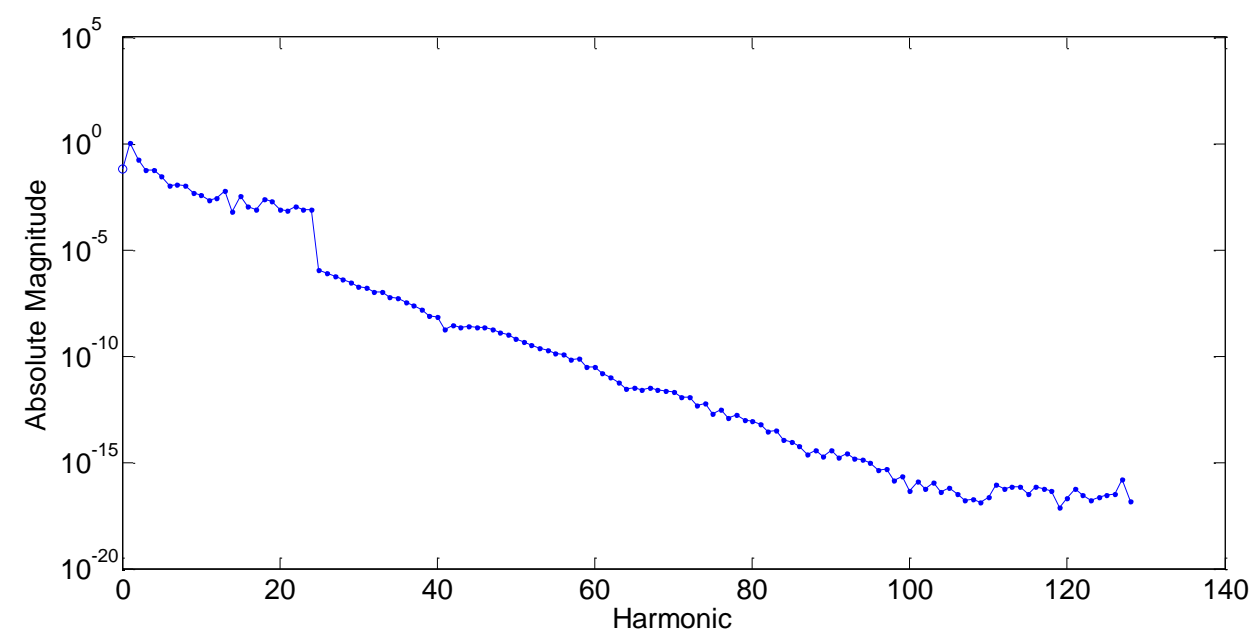

Figure 14. Absolute magnitudes of the total 128 response harmonics at turning point frequency ratio $\Omega_{1} / \omega_{n}=1.5269$ for oscillator with non-expansible stiffness and 24-harmonic forcing.

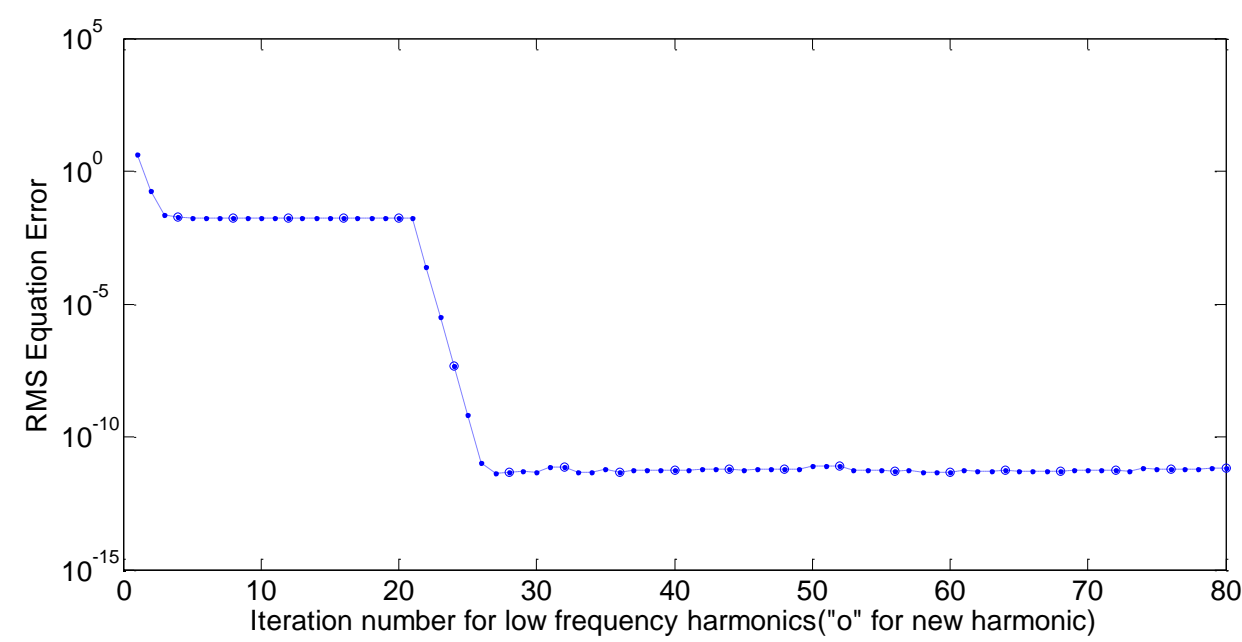

Figure 15. Equation error RMS at turning point frequency ratio $\Omega_{1} / \omega_{n}=1.5269$ for oscillator with nonexpansible stiffness and 24-harmonic forcing. 

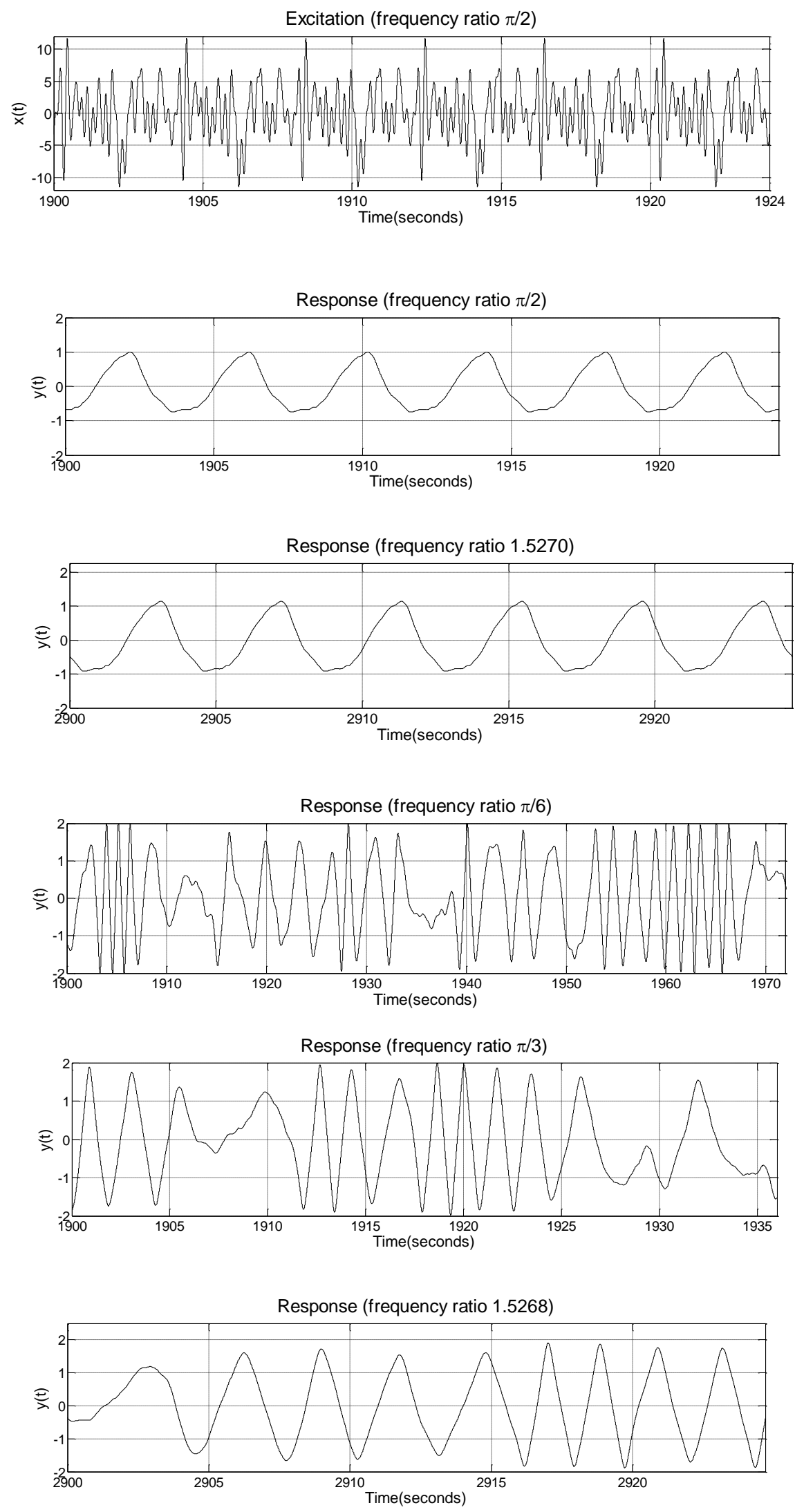

Figure 16. Periodic excitation and simulation response at specific frequency ratios. 